\title{
TITLE:
}

\section{Cytonuclear discordance and historical demography of two brown frogs, Rana tagoi and R. sakuraii (Amphibia: Ranidae).}

\section{$\operatorname{AUTHOR}(\mathrm{S})$ :}

Eto, Koshiro; Matsui, Masafumi

\section{CITATION:}

Eto, Koshiro ...[et al]. Cytonuclear discordance and historical demography of two brown frogs, Rana tagoi and R. sakuraii (Amphibia: Ranidae).. Molecular phylogenetics and evolution 2014, 79C: 231-239

\section{ISSUE DATE:}

2014-06-27

URL:

http://hdl.handle.net/2433/189320

\section{RIGHT:}

(c) 2014 Elsevier B.V.; この論文は出版社版でありません。引用の際には 出版社版をご確認ご利用ください。; This is not the published version. Please cite only the published version. 
1 Cytonuclear Discordance and Historical Demography of Two Brown Frogs, Rana tagoi

\section{and R. sakuraii (Amphibia: Ranidae)}

\section{KOSHIRO ETO and MASAFUMI MATSUI*}

\section{Graduate School of Human and Environmental Studies, Kyoto University, Yoshida}

Nihonmatsu-cho, Sakyoku, Kyoto 606-8501, Japan

*Corresponding author. Phone: +81-75-753-6846;

$$
\text { FAX: +81-75-753-6846; }
$$

E-mail: fumi@zoo.zool.kyoto-u.ac.jp

\section{Abstract}

Prior studies of mitochondrial genomic variation reveal that the Japanese brown frog Rana tagoi comprises a complex of cryptic species lineages, and that $R$. sakuraii arose from within this complex. Neither species forms a monophyletic group on the mitochondrial haplotype tree, precluding a simple explanation for the evolutionary origins of $R$. sakuraii. We present a more complete sampling of mitochondrial haplotypic variation (from the ND1 and 16S genes) plus DNA sequence variation for five nuclear loci (from the genes encoding NCX1, NFIA, POMC, SLC8A3, and TYR) to resolve the evolutionary histories of these species. We test hypotheses of population assignment (STRUCTURE) and isolation-with-migration (IM) using the more slowly evolving nuclear markers. These demographic analyses of nuclear genetic variation confirm species-level distinctness and integrity of $R$. sakuraii despite its apparent polyphyly on the mitochondrial haplotype tree. Divergence-time estimates from both the mitochondrial haplotypes and nuclear genomic markers suggest that $R$. sakuraii originated approximately one million years ago, and that incomplete sorting of mitochondrial haplotype lineages best explains 
non-monophyly of $R$. sakuraii mitochondrial haplotypes. Cytonuclear discordance elsewhere in R. tagoi reveals a case of mitochondrial introgression between two species lineages on Honshu. The earliest phylogenetic divergence within this species group occurred approximately four million years ago, followed by cladogenetic events in the Pliocene and early Pleistocene yielding 10-13 extant species lineages, including $R$. sakuraii as one of the youngest.

Key words: species complex; incomplete lineage sorting; introgression; isolation with migration

\section{Introduction}

Japanese brown frogs Rana tagoi and R. sakuraii are known to show a complicated genealogical relationship (Tanaka et al., 1996; Eto et al., 2012, 2013). Rana tagoi occurs widely on the main and peripheral islands of the Japanese archipelago except for Hokkaido and the Ryukyus. While most brown frogs breed in open, still waters, $R$. tagoi breeds in subterranean streams where the larvae can metamorphose without feeding (Matsui and Matsui, 1990; Maeda and Matsui, 1999). These distinctive traits might be the product of adaptation to the mountainous environments of the Japanese archipelago. Conversely, $R$. sakuraii, occurring only on Honshu sympatric with $R$. tagoi, breeds under rocks in open streams, and adult frogs have several characters suitable for a lotic environment (e.g., they possess fully developed toe webs, which are less well developed in $R$. tagoi), although its eggs and larvae share traits with those of R. tagoi. From these facts, Matsui and Matsui (1990) postulated that $R$. sakuraii speciated from a $R$. tagoi-like ancestor when it adapted to stream environments. This hypothesis is supported by phylogenetic analyses of mitochondrial haplotypes, in which $R$. sakuraii is embedded in $R$. tagoi lineages (Tanaka et al., 1996; Eto et al., 2012). However, neither of the species is monophyletic on the mitochondrial haplotype tree (Eto et al., 2012). Mitochondrial haplotype variation reveals that $R$. tagoi is divided into numerous species lineages, and some of these lineages are reproductively isolated from each other (Eto et al., 2012, 2013). As is clear from 
53 these studies, $R$. tagoi contains multiple cryptic species, one of which is the sister taxon to $R$.

54 sakuraii. Two hypotheses potentially explain polyphyly of $R$. sakuraii haplotypes on the mitochondrial haplotype phylogeny. Incomplete lineage sorting (ILS), retention of disparate haplotype lineages from an $R$. tagoi-like ancestor, is the simplest explanation if $R$. sakuraii originated very recently, within the past approximately one million years. Alternatively, introgression of mitochondrial haplotypes resulting from gene flow between $R$. sakuraii and a sympatric lineage of $R$. tagoi could explain the anomalous phylogenetic distribution of $R$. sakuraii mitochondrial haplotypes.

In this study, we analyse sequence data for two mitochondrial and five nuclear loci to test these hypothesis and to estimate divergence times and demographic patterns of these two species. Expanded sampling of mitochondrial haplotype variation relative to earlier studies yields increased precision of the mitochondrial phylogenetic analysis. We test hypotheses of population assignment (Pritchard et al, 2000) and isolation-with-migration (IM; Hey, 2010) using the more slowly evolving nuclear markers to verify inferences made from the mitochondrial haplotype phylogeny.

\section{Materials and Methods}

\subsection{Sampling strategy}

For each species, we chose samples belonging to representative localities/mt-lineages based on previous studies (e.g., Eto et al., 2012). We analysed 107 samples of $R$. tagoi (including three samples each of the subspecies R. t. yakushimensis and R. t. okiensis from peripheral islands) and 21 of $R$. sakuraii from 81 localities (Fig. 1, Table S1). To the mtDNA phylogenetic analysis, we added GenBank data for R. kobai (AB685768), R. sauteri (AB685767), R. tsushimensis (AB639592, AB639752), and R. ulma (AB685780) as outgroup taxa based on known phylogenetic relationships (Tanaka-Ueno et al., 1996, 1998). 


\subsection{Sequencing of DNA}

Total DNA was extracted from frozen or ethanol-preserved tissues using standard phenolchloroform extraction procedures. Then, we amplified fragments containing the target region (two mitochondrial genes, 16S ribosomal RNA [16S] and NADH dehydrogenase subunit 1 [ND1]; and five nuclear genes, sodium-calcium exchanger 1 [NCX1=SLC8A1], nuclear factor I/A [NFIA], pro-opiomelanocortin [POMC], sodium-calcium exchanger 3 [SLC8A3], and tyrosinase $[T Y R]$ ) by polymerase chain reaction (PCR). The experimental conditions and PCR techniques were essentially identical to those reported previously (Eto et al., 2012). The amplified PCR products were purified by polyethylene glycol (PEG) precipitation. The cycle sequence reactions were performed out with an ABI PRISM Big Dye Terminator ver. 3.1 Cycle sequencing Kit (Applied Biosystems) and sequenced on an ABI 3130 automated sequencer. We used the primers listed in Table S2 for PCR and sequencing, and all samples/loci were sequenced in both directions.

\subsection{Alignment of DNA, haplotype determination, and data characteristics}

Sequence alignment was conducted using MUSCLE (Edgar, 2004). For heterozygous nuclear genes, we used PHASE ver. 2.1 (Stephens et al., 2001) to determine haplotypes. In this analysis, the threshold of probability was set to small values (0.5-0.6) following Garrick et al. (2010). Before analysing the historical demography, we also used IMgc (Woerner et al., 2007) to detect the largest non-recombining block of nDNA for IM analysis, because IMa2 assumes no intra-locus recombination (Hey and Nielsen, 2004). As data parameters, we calculated the summary statistics of variable sites $(v s)$, number of haplotypes $(h)$, haplotype diversity $\left(H_{d}\right)$, and nucleotide diversity $(\pi)$. We also checked the neutrality of the five nuclear loci with Tajima's $D$ (Tajima, 1989). Since none of them showed significant deviation from zero (Table S3), these loci were considered neutral markers. We conducted all of these calculations using DnaSP (Rozas et al., 2003). 


\subsection{Population assignment based on mtDNA}

A phylogenetic analysis was conducted using the two mitochondrial genes. First, we selected the best substitution model for each gene using Kakusan4 (Tanabe, 2011) based on the Akaike information criterion (AIC). Then, phylogenetic trees based on the maximum-likelihood method (ML) and Bayesian inference (BI) were constructed using TREEFINDER ver. Mar. 2011 (Jobb, 2011) and MrBayes ver. 3.2.1 (Ronquist and Huelsenbeck, 2003), respectively. For

112 the ML tree, we conducted non-parametric bootstrap analysis with 1000 replicates, and

113 branches with a bootstrap value (BS) of 70\% or greater were regarded as significantly supported.

114 In the BI analysis, two independent runs of four Markov chains were conducted for 10 million generations (sampling frequency one tree per 100 generations); the first three million generations were discarded as burn-in. Convergence of parameters was checked using Tracer ver. 1.5 (Rambaut and Drummond, 2009). We considered a Bayesian posterior probability (BPP) of 0.95 or greater as significant support. From the results of both analyses, we used mitochondrial haplotype clades, levels of haplotype divergence, and geographic distributions to diagnose hypothetical species lineages, which were treated as population units based on mtDNA in the later analyses.

\subsection{Population assignment based on $n D N A$}

Rana tagoi and $R$. sakuraii are so close genetically as to cause difficulty constructing phylogenetic trees using nDNA sequences (Eto et al., 2012, 2013). Therefore, we conducted clustering analysis using STRUCTURE ver. 2.3.3 (Pritchard et al., 2000) to delimit population units based on nDNA. We applied an admixture and allele-frequency-independent model to

128 haplotype data for the nuclear loci, and calculated 500,000 generations following 100,000

129 generations of burn-in. The number of clusters $(K)$ was set from 1 to 10 , and 10 independent

130 iterations were conducted for each $K$. The most likely $K$ was determined by the likelihood 
131 distribution of each iteration and the delta $K$ value (Evanno et al., 2005). We also constructed

132 haplotype networks for each gene based on the median-joining method using Network ver. 4.6

133 (Bandelt et al., 1999) to examine the relationships among nuclear haplotypes.

134

135

136

137

138

139

140

141

142

143

144

145

146

147

148

149

150

151

152

153

154

155

156

\subsection{Divergence dating based on mtDNA}

To estimate the divergence time between mt-lineages, we conducted Bayesian analysis using BEAST ver. 1.7.5 (Drummond et al., 2012). For each calibration, 10 million generations of run (of which the first three million were discarded as burn-in) were conducted under a nonautocorrelated log-normal relaxed clock model. Tracer ver. 1.5 (Rambaut and Drummond, 2009) was used to check the parameter distributions and effective sample size. We applied the following two different calibrations:

Calibration I: The molecular evolutionary rate of $1.38 \%$ (0.69\% per lineage) per MY was applied. This value was estimated for the ND1 and ND2 regions of Bufo (Macey et al., 1998), and only ND1 data were used in this calculation. The evolutionary rate of this region is similar among a wide range of vertebrates (Macey et al., 2001). We thus used that rate, despite considerable phylogenetic distance between Rana and Bufo.

Calibration II: Using only $16 S$ data, we applied the evolutionary rate of $0.66 \%$ ( $0.33 \%$ per lineage) per MY estimated for 16S of Leiopelma (Fouquet et al., 2009).

\subsection{Estimation of historical demography}

The historical demography, especially the patterns of gene flow and divergence times among species or genetic groups, was examined using coalescent analysis with the Bayesian IM model. We analysed the nDNA data using the program IMa2 (Hey 2010), and estimated the effective population size, $N_{e}$, population migration rate, $2 N_{e} M$, and population divergence time, T. As the mutation rate of nuclear genes, we applied $0.047 \%$ per MY per lineage for NCX1 (reported in the genus Hydromantes; Rovito 2010), 0.072\% (0.061-0.083\%) for POMC 
157 (Hyperolius; Lawson, 2010), and 0.047\% (0.027-0.067\%) for SLC8A3 (amphibians in general;

158 Roelants et al., 2007). The geometric mean of these values, approximately $2.71 \times 10^{-7}$ mutations

159 per year per locus, was used as the mutation rate $(\mu)$ to scale each demographic parameter.

160 Based on several test runs, the upper bounds for the parameters were set at $\theta=10-20, t=3-5$,

161 and $m=10-25$, and five million steps (sampling frequency one tree per 50 steps) of

162 calculations were performed for 30 heated chains after two million burn-in steps. We conducted

163 three independent runs, and finally combined the results using the L-mode option of IMa2.

164 Since $R$. tagoi and $R$. sakuraii typically start to breed at the age of 3 years (Kusano et al., 1995a,

165 b), we applied this value as the generation time of the two species. The trendline plots and

166 effective sample sizes were monitored to ensure good mixing and convergence of parameters.

167 The significance of $2 N_{e} M$ was determined using the log-likelihood ratio (LLR) test of

168 Nielsen and Wakeley (2001). We also used the parameter comparison option (with the -p6

169 command) of IMa2 and output the list of probability, which indicates one parameter to be

170 greater than the other. The relative strength of genetic isolation was evaluated using $2 N_{e} M$

171 values (strong [ $\left.2 N_{e} M \leq 1\right]$, moderate $\left[1<2 N_{e} M \leq 5\right]$, and weak $\left[5<2 N_{e} M \leq 25\right]$ : Wright, 1931;

172 Waples and Gaggiotti, 2006; Reilly et al., 2012).

173

174 3. Results

175 3.1. Sequence characteristics

$176 \quad$ We obtained complete mitochondrial 16S (1612bp) and ND1 (967bp) sequences for all

177 samples. There were 489 parsimoniously informative sites within the ingroup: 244 for $16 S$ and

178245 for ND1. The other statistics are listed in Table S3.

179 In the sequences of the five nuclear loci for all 128 samples, only POMC had in-dels, and

180 these sites were omitted from the subsequent analyses. For haplotype determination using

181 PHASE, all haplotypes in all samples/loci were determined successfully, except for one sample

182 for POMC and two for TYR, which were treated as null alleles in subsequent analyses. The 
183 sequence length and statistics of each locus are listed in Table S3. Overall, each parameter

184 generally indicated great genetic diversity in $R$. tagoi and $R$. sakuraii. Of the five nuclear loci,

185

186

187

188

189

190

191

192

193

194

195

196

197

198

199

200

201

202

203

204

205

206

207

208 TYR was the most variable ( $H_{d}=0.955$ and $\pi=0.017$ for all samples) and NFIA was the least variable (0.735 and 0.003, respectively).

\subsection{Population assignment: Mitochondrial DNA results}

The best substitution model selected in the ML analysis was the general time reversible (GTR; Tavaré, 1986) model with the optimized gamma shape parameter (G) of 0.158 and the proposition of invariable sites (I) of 0.144 for $16 S$ and the J1 (Jobb, 2011) model + G (0.543) + I (0.312) for ND1. For BI, the models were GTR + G (0.082) + I (0.226) and GTR + G (0.892) + I (0.226) for $16 S$ and $N D 1$, respectively. The constructed ML $(-\ln L=15500.618)$ and BI (15863.190) trees were essentially identical in topology, and only the ML tree is shown in Fig. 2. We followed Eto et al. (2012) for the names of each genetic group.

The phylogenetic relationships obtained were fundamentally identical to those reported by Eto et al. (2012). The ingroup was divided into two large haplotype clades (A and B), and both of these included subclades judged by their geographic distributions to diagnose separate species lineages (A-1ab to A-9abc and B-1 to B-2ab); Clade B (ML-BS = 82\% and BPP = 1.00) contained only haplotypes from $R$. tagoi, while Clade A (ML-BS = 93\% and $\mathrm{BPP}=1.00)$ included both $R$. tagoi and $R$. sakuraii haplotypes. Each clade/lineage was well supported (ML-BS $\geqq 70 \%$, BPP $\geqq 0.95$ ). The statistical support for nodes was generally better than in the previous study, and more detailed phylogenetic relationships were clarified, particularly those among the lineages in Clade A. In Clade A, the lineages from Honshu Island (A-1ab to A-6) formed a subclade (A' in Fig. 2. ML-BS = 73\% and BPP = 0.98) against the Shikoku and Kyushu subclade (A"; ML-BS = 79\% and BPP = 0.95). Within Subclade A', three additional lineage groups were recognised: one consisted of Lineages A-1a and A-1b (MLBS $=82 \%$ and BPP = 1.00); the second of Lineages A-2 and A-3 (ML-BS $=70 \%$ and 
$\mathrm{BPP}=0.98)$; and the third Lineages A-4, A-5, and A-6 (ML-BS = 79\% and BPP = 1.00). The haplotypes obtained from $R$. sakuraii were included in Lineages A-2 and A-3. Lineage A-2 also contained $R$. tagoi haplotypes, although haplotypes were not shared between the two species.

\subsection{Population assignment: Nuclear DNA results}

The results of the clustering analysis using STRUCTURE are shown in Fig. 3. For all samples, $K=2$ was supported by the test of delta $K$, and two clusters (I and II) were recognised. Almost all samples were clearly assigned to each cluster (posterior probabilities $\geq 80 \%$ ), indicating strong genetic isolation between the two nDNA clusters. Although the division of the two nuclear clusters (I and II) did not completely correspond to that of the two mitochondrial clades (A and B), Cluster II was largely concordant with mitochondrial Subclade A', with the exception of Lineage A-1a (Fig. 3). Clusters I and II also were separated on the haplotype networks of some nuclear genes (e.g., NCX1, NFIA, and SLC8A3; Fig. S1). However, in relatively more variable genes like $T Y R$, the haplotype relationships were highly complex and their separation was not clear (Fig. S1). Furthermore, haplotypes were more or less shared between Clusters I and II in all loci, indicating ILS in these nuclear genes.

Since the two large clusters seemed to contain several subclusters, we independently reanalysed samples for the two clusters. Within Cluster I, the population assignment with $K=2$ was supported (Fig. 3). In this clustering, the division of subclusters was still roughly correlated with the mt-lineages: the lineages from the main islands (A-1a, A-7, A-9a, and B-2ab) tended to form a subcluster and the lineages from the peripheral islands (A-8, A-9c, and B-1) formed another. One lineage, A-9b, included samples assigned to both of these subclusters. Except for Lineage A-9b, samples of the two subclusters were clearly assigned to either subcluster. In contrast, $K=3$ was supported within Cluster II using the delta $K$ test and likelihood distribution. In this division, $R$. tagoi Lineages A-1b and A-4, R. tagoi A-2, and R. sakuraii (A-3 and part of A-2) each formed a subcluster (Fig. 3). The separation of these subclusters was clear (posterior 
235 probabilities $>80 \%$ ), with a few exceptional samples in the $R$. sakuraii subcluster. By contrast, 236 many samples of lineages A-5 and A-6 were not clearly assigned to particular subclusters, and 237 showed intermediate genetic structures between $R$. tagoi of A-2 and R. sakuraii.

238

239 and Whitlock 2004).

\subsection{Divergence times of the mitochondrial lineages}

The results of divergence dating for the major nodes on the mitochondrial genealogy are listed in Table 1. Although we applied the evolutionary rates of phylogenetically remote taxa (Bufo and Leiopelma) the divergence times obtained for the ingroup were similar in the two calibrations. Two major mt-clades (A and B: node 1 in Fig. 2) were estimated to have diverged 4.2-4.0 (95\% highest posterior density interval [HPD] of 6.2-2.3) MYA. Then Subclades A' and A" (node 2) split 2.8-2.6 (4.1-1.6) MYA, followed by the separation within Clade B (node 22) 2.7-2.3 (4.3-1.2) MYA. The two lineages including $R$. sakuraii samples, A-2 and A-3, separated from each other 2.1-1.9 (3.1-1.1) MYA (node 7), followed by internal divergence during 1.4-0.9 (2.2-0.4) MYA (nodes 8 and 9). The most recently divergent lineages were B-2a and B-2b (node 23), which split at 1.4 (2.2-0.7) MYA. These estimates indicate that the divergence of each major mitochondrial clade/lineage began in the mid-to-late Pliocene and was approaching completion in the mid Pleistocene.

\subsection{Historical demography}

As shown above, the results of the population assignment were not completely concordant between mt- and n-DNA (Figs. 2 and 3). In estimating demographic parameters, we used only nDNA data because nuclear markers are thought to be more conservative than mitochondrial ones, which are more likely to be affected by introgression than the nuclear markers (Ballard 
260

261

262

263

264

265

266

267

268

269

270

271

272

273

274

275

276

277

278

279

280

281

282

283

284

285

\subsubsection{Historical demography between Clusters I and II}

First, we conducted a coalescent analysis using IMa2 for the two large nuclear clusters: I and II. Each parameter showed single peaks in their probability density distributions (Fig. S2). The parameter values obtained are listed in Table 2 . The estimated population migration rate $\left(2 N_{e} M\right)$ for I to II (I $\rightarrow$ II) was $0.52(0.24-1.12)$. In the opposite direction; i.e. II $\rightarrow$ I, the parameter value tended to be larger, with $2 N_{e} M_{\text {II } \rightarrow \text { I }}$ being $1.23(0.70-2.14)$. The LLR test showed that all of these values were significantly larger than zero $(p<0.01)$, suggesting that clusters I and II have maintained a degree of gene flow after their divergence. However, strong to moderate genetic isolation would exist between the two clusters because the $2 N_{e} M$ values obtained were relatively small (ca. 1 or smaller: Wright, 1931; Waples and Gaggiotti, 2006; Reilly et al., 2012). The effective population size estimated for I, II, and their ancestor was 2.2 (1.7-2.9), 1.7 (1.3-2.3), and 0.4 (0.2-0.8) million individuals, respectively. The ancestral population size was smaller than those at present, as supported by parameter comparison of $\theta$ (the posterior probabilities were 1.00 for each comparison). The population size of II tended to be smaller than that of I, but the tendency was not supported statistically (BPP $<0.95$ ). The population divergence time (T) of I and II was estimated as 2.7 (4.4-2.2) MYA. Although its 95\%HPD was relatively wide, this estimate was younger than the divergence time of the two major mt-clades (A/B; ca. 4.2-4.0 MYA), but almost equal to those of A'/A" (ca. 2.8-2.6 MYA) and B-1/B-2 (ca. 2.7-2.3 MYA) (Table 1).

\subsubsection{Historical demography between R. tagoi and R. sakuraii}

Then, we compared demographic parameters between $R$. tagoi and R. sakuraii. As R. tagoi (Rt), we chose mt-Lineages A-2, 5, and 6, which were genetically close to $R$. sakuraii (Rs) in the mitochondrial and nuclear DNA analyses, as shown above (see Figs. 2 and 3). Since our dataset was not sufficiently informative to analyse a four-populations model, we combined Lineages A-5 and A-6 as a single group; these showed close genetic relationships in both 
286

287

288

289

290

291

292

293

294

295

296

297

298

299

300

301

302

303

304

305

306

307

308

309

310

mitochondrial and nuclear analyses (Figs. 2, 3). We conducted two separate analyses under different population schemes: (1) three-populations model, in which $R$. sakuraii $(R s)$ and $R$. tagoi $(R t)$ Lineage A-2 were assumed to be mutually close compared to A-5 and 6, based on the mtDNA genealogy, and (2) two-populations model based on the current classification (Rs vs. $R t$ A-2, 5, and 6).

In the three-populations model, significant gene flow ( $p<0.05$ in the LLR test) was detected only in $R$. tagoi A-5+6 $\rightarrow$ A-2 $\left(2 N_{e} M_{R t}\right.$ A-5+6 $\rightarrow R t$ A-2 was 3.79 [0.75-9.50]; Fig. S3 and Table 2) and A-5+6 $\rightarrow R$. sakuraii ( $2 N_{e} M_{R t} \mathrm{~A}-5+6 \rightarrow R s$ was 0.40 [0.04-2.00]), and no significant gene flow was recognized between $R$. sakuraii and $R$. tagoi A-2 $(p>0.05)$. These results indicated that the genetic isolation between $R$. tagoi A-2 and A-5+6 was moderate $\left(1<2 N_{e} M \leq\right.$ 5), but the gene flow was strongly biased to one direction (from A-5+6 to A-2). Although gene flow existed between the two species, the direction was limited (R. tagoi A-5+6 $\rightarrow R$. sakuraii), and the population migration rate obtained was small $\left(2 N_{e} M \leq 1\right)$, indicating strong genetic isolation between $R$. sakuraii and $R$. tagoi lineages. The estimated effective population size (a million individuals) was similar between $R$. tagoi A-2 (0.80 [0.34-2.06]) and A-5+6 (0.79 [0.38-1.76]), but was smaller in R. sakuraii (0.16 [0.07-0.32]). This tendency was supported in the statistical test, in which $N_{e}$ for $R$. sakuraii was significantly smaller than those for $R$. tagoi lineages $(\mathrm{BPP}>0.95)$.

We could not obtain a sufficient estimate for gene flow between the ancestral populations because no obvious peaks of probability for the parameter $2 N_{e} M$ were recognised (Table 2). The estimated ancestral population size $\left(N_{e}\right)$ was $0.21(0.01-4.06)$ for $R$. sakuraii $+R$. tagoi A-2, and was $0.43(0.23-0.77)$ for the common ancestor of $R$. sakuraii, $R$. tagoi A-2 and A-5+6. The estimated $N_{e}$ for the ancestors tended to be smaller than the present $N_{e}$ for $R$. tagoi (A-2, A-5+6) and larger than that for R. sakuraii, but the tendencies were not supported statistically (BPP < 0.95). The time of population divergence estimated for $R$. sakuraii/R. tagoi A-2 (1.1 [2.3-0.6] 
311 MYA) was much younger than that for the ancestors (2.15 [6.11-1.31] MYA), although the

312 credibility intervals largely overlapped.

313 In the two-populations model, significant gene flow from $R$. tagoi to $R$. sakuraii was again

314 detected (2NeM $M_{R t \rightarrow R s}$ was 0.51 [0.14-1.17]: Fig. S3 and Table 2), but such trend was not

315 recognized in the opposite direction (Fig. S3 and Table 2). These results indicate strong to medium isolation between the two species, although small and unidirectional gene flow exists. The $2 N_{e} M$ value for $R$. tagoi $\rightarrow R$. sakuraii in this model was similar to the value for $R$. tagoi A5+6 $\rightarrow R$. sakuraii in the three-populations model shown above (Table 2).

The estimated $N_{e}$ showed values and tendencies similar to those obtained in the threepopulations model; $N_{e}$ for $R$. sakuraii $(0.17$ [0.09-0.34]) was significantly smaller (BPP > 0.95) than that of $R$. tagoi (1.61 [0.99-2.65]). The estimates for ancestral $N_{e}(0.37[0.10-0.68]$ in the two-populations model) also are similar between the models. The divergence time estimated for the two species, 1.2 (2.9-0.6) MYA, was slightly older than that estimated by the threepopulation model (ca. 1.1 MYA).

\section{Discussion}

4.1. Discordance between the classification and patterns of genetic variation using different markers

Our new data and analyses confirmed the major patterns of mitochondrial genomic variation reported previously (Eto et al., 2012). Mitochondrial haplotypes obtained from $R$. sakuraii were genealogically embedded in those from $R$. tagoi, and neither species was monophyletic on the haplotype tree. The mitochondrial and nuclear data considered together indicate that $R$. sakuraii constitutes a single species lineage. Rana sakuraii corresponds largely to Lineage A-3 on the mitochondrial haplotype tree (Fig. 2), with its sister lineage being $R$. tagoi populations bearing 
mitochondrial haplotypes. We examine the hypotheses of incomplete lineage sorting and gene flow as possible explanations for this pattern. The following three scenarios could explain the phylogenetic pattern of mitochondrial haplotypes of lineages A-2 and A-3 (Fig. 2): (1) recent speciation of $R$. sakuraii from $R$. tagoi Lineage A-2, which led to ILS of mtDNA at the species level; (2a) past mitochondrial introgression from R. tagoi A-2 to R. sakuraii; and (2b) introgression in the opposite direction (Fig. 4). If recent separation of $R$. sakuraii from $R$. tagoi A-2 was the case, the ILS hypothesis (1) would be the simplest explanation. However, if the speciation was shown to be old, especially much older than the divergence time within mtLineage A-2, this hypothesis would be rejected. Conversely, the past-introgression hypotheses (2) would be applicable if the speciation of the two species coincided with the split between Lineages A-2 and A-3 (2a), or the separation of these two lineages from the others (2b).

Detection of historical gene flow between $R$. sakuraii and $R$. tagoi A-2 for the nuclear markers also would support the past introgression hypotheses.

The genetic relationship based on the STRUCTURE analysis using nDNA was discordant with the mitochondrial genealogy, and $R$. sakuraii and $R$. tagoi A-2 tended to be separated in different subclusters (Fig. 3). This result likely reflects their heterospecific status. The demographic analysis using IMa2 showed that the separation of $R$. sakuraii from $R$. tagoi lineages (ca. 1.1 MYA and 1.2 MYA in three- and two-populations models, respectively; Table 2) was younger than the separation of mt-Lineages A-2 and A-3 (ca. 2.1-1.9 MYA; Table 1), and was similar to the divergence within these lineages (ca. 1.4-0.9 MYA). The date of speciation would correspond to, or be younger than, the population divergence time estimated by IMa in this case. So these results favour the ILS hypothesis, although the credibility intervals of these estimates overlapped.

Based on the genealogy obtained (Fig. 2), mitochondrial introgression between $R$. tagoi A-2 and $R$. sakuraii happened several times if the hypotheses 2 were the case (for example, two independent introgression events should be presumed in the hypothesis 2a). Thus the 
363

364

365

366

367

368

369

370

371

372

373

374

375

376

377

378

379

380

381

382

383

384

385

386

387

388

introgression hypotheses assume rampant hybridization of $R$. tagoi A-2 and $R$. sakuraii in the past. The IM analyses based on two different models showed gene flow from $R$. tagoi to $R$. sakuraii. However, this unidirectional gene flow seems to depend largely on the flow from $R$. tagoi A-5+6 to R. sakuraii, because no significant flow between $R$. tagoi A-2 and R. sakuraii was detected (Table 2). These results do not support rampant hybridization of $R$. tagoi A-2 and $R$. sakuraii, even though inter-specific gene flow did exist. From these considerations, the ILS hypothesis would be more plausible than the introgression hypothesis to explain the relationships of the two species on the mitochondrial genealogy.

The estimated time of the split of $R$. sakuraii and $R$. tagoi Lineage A-2 (ca. 1.2-1.1 MYA) is younger than those of other Japanese frogs (e.g., ca. 2.3 MYA between Odorrana ishikawae/O. splendida and ca. 1.7 MYA between O. amamiensis/O. narina [Matsui et al., 2005]; and around 5.7-4.0 MYA among Bufo torrenticola and two subspecies of B. japonicus [Igawa et al., 2006]), and seems to have occurred after the rough formation of the Japanese archipelago (see the next section). Although the ILS of mtDNA at the species level is relatively rare because of its small effective number of gene copies, it occurs occasionally in some situations, such as speciation within the past millions years. It could be applicable in the case of $R$. sakuraii and R. tagoi, because their speciation is estimated to be only about one million year ago. Rana sakuraii has several traits adaptive to stream breeding in contrast to the subterranean breeding $R$. tagoi, although they share many other characters (Matsui and Matsui, 1990). It suggests that the speciation of $R$. sakuraii was triggered by adaptation to a new breeding habitat, which is a process that often promotes rapid speciation (Coyne and Orr, 2004).

\subsection{Evolutionary history of the two species}

Rana tagoi and $R$. sakuraii are endemic to the Japanese archipelago and no close relatives are known from the continent, although $R$. sauteri, a lotic breeding brown frog from Taiwan, is thought to be their sister lineage (Tanaka-Ueno et al., 1998). Our data do not contradict with this idea (Fig. 1). Since the continental allies of R. sauteri are also unknown, the dispersal route of 
the ancestor of the $R$. tagoi complex to the Japanese mainland is uncertain. The estimated time of separation of $R$. sauteri and $R$. tagoi complex varies between the calibrations (22.0-11.6 MYA; Table 1), but around the early to middle Miocene. In this period the opening of the Japan Sea began (Iijima and Tada, 1990), although the Japanese and Ryukyu archipelagos, as well as Taiwan were not yet isolated from the Eurasian continent (Chinzei and Machida, 2001). Therefore the common ancestor of $R$. sauteri and the $R$. tagoi complex would have been distributed in the continental areas corresponding to the present Japanese archipelago to Taiwan, but the ancestral allies would have been extinguished thereafter on the continent and the Ryukyus, leaving relict species in Japan and Taiwan.

In any case, the ancestral population of the $R$. tagoi complex is thought to have diverged into two major clades, A and B (Fig. 2), in the mid Pliocene (ca. 4.2-4.0 MYA). The ancient Japanese archipelago was already roughly formed by the late Miocene (Chinzei and Machida, 2001), and the separation of lineages ancestral to the clades is thought to have occurred on the archipelago. The ancestor at this period would have been a $R$. tagoi-like subterranean breeder because all of the present genetic groups of the two species have a common larval trait (e.g., no need to feed until metamorphosis) thought to be adapted to such an environment.

Then, the divergence within Clade A occurred in the late Pliocene (ca. 2.8-2.6 MYA), separating populations on or near Honshu from ones on or near from Kyushu and Shikoku.

407 Cluster II as identified by nuclear markers (Fig. 3) is equivalent to populations diagnosed by 408 mitochondrial haplotype Subclade A' excluding Lineage A-1a. Lineages of Subclade A' occur on Honshu, whereas those of mitochondrial Subclade A" are associated with Kyushu or Shikoku.

410 Because populations of mitochondrial haplotype Clade B also occur on Honshu, Honshu is

411 likely the ancestral source of this species complex, and expansion to the ancestral areas of

412 Kyushu and Shikoku likely produced the major cladogenetic event within mitochondrial Clade

413 A. Approximately 1.8-1.4 MYA (the divergence time estimated for mitochondrial haplotypes

414 of Lineages A-1a and A-1b), introgression of mitochondrial haplotypes from a population in 
415 mitochondrial Subclade A' to one in Clade B produced the anomalous result that Lineage A-1a

416 appears in an incorrect position on the mitochondrial haplotype tree. The best interpretation is

417 that Lineage A-1a is closest phylogenetically to the lineages of mitochondrial Clade B as

418 revealed by the nuclear markers, in contrast to its position on the mitochondrial haplotype tree.

419 Occurrence of Subclade A' and Clade B in geographic proximity on Honshu further supports

420 this interpretation. The divergences within SubcladesA', A", and Clade B started around 2.7-2.3

421 MYA, and splitting of the major mt-lineages was roughly completed by the middle Pleistocene

422 (around 1.4 MYA). In this period, the populations on peripheral islands were isolated

423 geographically, and some survived and evolved into the extant subspecies; i.e., R. .

424 yakushimensis of Lineage A-8 and R.t. okiensis of B-1.

425 The estimated date of speciation of R. sakuraii was younger than the formation of the major 426 population lineages discussed above. Rana sakuraii would have originated ca. 1.2-1.1 MYA

427 based on the IM analysis (Table 2), likely in association with the adaptation to a new breeding 428 environment as discussed above. The effective population size of $R$. sakuraii (ca. 0.2 million 429 individuals) is smaller than that of the closest mt-lineage of $R$. tagoi (ca. 0.8 million individuals 430 for Lineage A-2), and suggests that a small ancestral population adapted to stream breeding led 431 to R. sakuraii.

432

433 5. Conclusion

$434 \quad$ Our data reveal that $R$. tagoi comprises multiple species lineages, which form a paraphyletic 435 group with respect to $R$. sakuraii. Because $R$. sakuraii arose only about one million years ago, incomplete lineage sorting of mitochondrial haplotypes best explains non-monophyly of $R$. sakuraii on the mitochondrial haplotype tree. Our study illustrates how mitochondrial haplotype 438 phylogenies combined with multilocus demographic analyses of nuclear haplotypes permits 439 precise resolution of species lineages and their genetic interactions. 
441

442

443

444

445

446

447

448

449

450

451

452

453

454

455

456

457

458

459

460

461

462

463

464

465

466

\section{Acknowledgements}

We would like to thank G. Aoki, K. Araya, H. Fujita, T. Hayashi, A. Hamidy, T. Hikida, M. Kato, Y. Kawahara, K. Kawauchi, Y. Kokuryo, N. Kuraishi, N. Maeda, Mm, Matsui, T. Matsuki, T. Matsuo, Y. Misawa, Y. Miyagata, A. Mori, S. Mori, N. Nakahama, T. Nakano, Y. Nakase, K. Nishikawa, S. Okada, J. Oki, M. Sakamoto, T. Shimada, T. Sugahara, T. Sugihara, H. Takeuchi, S. Tanabe, A. Tominaga, T. Ueno, M. Yamagami, Y. Yamane, Y. Yamazaki, N. Yoshikawa, and everyone else who helped us in collecting specimens. KE thanks N. Kuraishi and N.

Yoshikawa for assisting laboratory works. We are also grateful to A. Larson and anonymous reviewers for valuable comments on the manuscript. This work was partly supported by grants from the Ministry of Education, Science and Culture, Japan (Nos. 07454234, 20405013 and 23405014) to MM.

\section{References}

Ballard, J.W.O., Whitlock, M.C., 2004. The incomplete natural history of mitochondria. Mol. Ecol. 13, 729-744.

Bandelt, H., Forster, P., Röhl, A., 1999. Median-joining networks for inferring intraspecific phylogenies. Mol. Biol. Evol. 16, 37-48.

Chinzei, K., Machida, H., 2001. Formation history of structural landforms and tectonic landforms in Japan, in: Yonekura, N., Kaizuka, S., Nogami, M., Chinzei, K. (Eds.), Regional Geomorphology of the Japanese Islands, Introduction to Japanese Geomorphology, Vol. 1. University of Tokyo Press, Tokyo, pp. 298-311.

Coyne, J.A., Orr, H.A., 2004. Speciation. Sinauer Associates, Sunderland, MA.

Drummond, A.J., Suchard, M.A., Xie, D., Rambaut, A., 2012. Bayesian phylogenetics with BEAUti and the BEAST 1.7. Mol. Biol. Evol. 29, 1969-1973.

Edgar, R., 2004. MUSCLE: multiple sequence alignment with high accuracy and high throughput. Nucleic Acids Res. 32, 1792-1797. 
467

468

469

470

471

472

473

474

475

476

477

478

479

480

481

482

483

484

485

486

487

488

489

490

491

Eto, K., Matsui, M., Sugahara, T., 2013. Discordance between mitochondrial DNA genealogy and nuclear DNA genetic structure in the two morphotypes of Rana tagoi tagoi (Amphibia: Anura: Ranidae) in the Kinki Region, Japan. Zool. Sci. 30, 553-558.

Eto, K., Matsui, M., Sugahara, T., Tanaka-Ueno, T., 2012. Highly complex mitochondrial DNA genealogy in an endemic Japanese subterranean breeding brown frog Rana tagoi (Amphibia, Anura, Ranidae). Zool. Sci. 29, 662-671.

Evanno, G., Regnaut, S., Goudet, J., 2005. Detecting the number of clusters of individuals using the software STRUCTURE: a simulation study. Mol. Ecol. 14, 2611-2620.

Fouquet, A., Green, D.M., Waldman, B., Bowsher, J.H., McBride, K.P., Gemmell, N.J., 2010. Phylogeography of Leiopelma hochstetteri reveals strong genetic structure and suggests new conservation priorities. Conserv. Genet. 11, 907-919.

Garrick, R.C., Sunnucks, P., Dyer, R.J., 2010. Nuclear gene phylogeography using PHASE: dealing with unresolved genotypes, lost alleles, and systematic bias in parameter estimation. BMC Evol. Biol. 10, 118.

Hey, J., 2010. Isolation with migration models for more than two populations. Mol. Biol. Evol. 27, 905-920.

Hey, J., Nielsen, R., 2004. Multilocus methods for estimating population sizes, migration rates and divergence time, with applications to the divergence of Drosophila pseudoobscura and D. persimilis. Genetics 167, 747-760.

Hurt, C., Silliman, K., Anker, A., Knowlton, N., 2013. Ecological speciation in anemoneassociated snapping shrimps (Alpheus armatus species complex). Mol. Ecol. 22, 45324548.

Igawa, T., Kurabayashi, A., Nishioka, M., Sumida, M., 2006. Molecular phylogenetic relationship of toads distributed in the Far East and Europe inferred from the nucleotide sequences of mitochondrial DNA genes. Mol. Phylogenet. Evol. 38, 250-260. 
492 Iijima, A., Tada, R., 1990. Evolution of tertiary sedimentary basins of Japan in reference to opening of the Japan Sea. J. Fac. Sci. Univ. Tokyo, Sect. II 22, 121-171.

494

495

496

497

498

499

500

501

502

503

504

505

506

507

508

509

510

511

512

513

514

515

516

517

Jacobsen, F., Omland, K.E., 2012. Extensive introgressive hybridization within the northern oriole group (Genus Icterus) revealed by three-species isolation with migration analysis. Ecol. Evol. 2, 2413-2429.

Jobb, G., 2011. TREEFINDER version March 2011. <http://www.treefinder.de>.

Kusano, T., Fukuyama, K., Miyashita, N., 1995a. Age determination of the stream frog, Rana sakuraii, by skeletochronology. J. Herpetol. 29, 625-628.

Kusano, T., Fukuyama, K., Miyashita, N., 1995b. Body size and age determination brown frog Rana tagoi tagoi by skeletochronology in southwestern Kanto. Jpn. J. Herpetol. 16, 29-34.

Lawson, L.P., 2010. The discordance of diversification: evolution in the tropical-montane frogs of the Eastern Arc Mountains of Tanzania. Mol. Ecol. 19, 4046-4060.

Macey, J.R., Schulte, J.A, Larson, A., Fang, Z., Wang, Y., Tuniyev, B.S., Papenfuss, T.J., 1998. Phylogenetic relationships of toads in the Bufo bufo species group from the eastern escarpment of the Tibetan Plateau: a case of vicariance and dispersal. Mol. Phylogenet. Evol. 9, 80-87.

Macey, J.R., Strasburg, J.L., Brisson, J. a, Vredenburg, V.T., Jennings, M., Larson, A., 2001. Molecular phylogenetics of western North American frogs of the Rana boylii species group. Mol. Phylogenet. Evol. 19, 131-143.

Maeda, N., Matsui, M., 1999. Frogs and Toads of Japan, Revised Edition. Bun-ichi Sogo Shuppan, Tokyo.

Matsui, M., 2011. On the brown frogs from the Ryukyu Archipelago, Japan, with descriptions of two new species (Amphibia, Anura). Curr. Herpetol. 30, 111-128.

Matsui, M., Shimada, T., Ota, H., Tanaka-Ueno, T., 2005. Multiple invasions of the Ryukyu Archipelago by Oriental frogs of the subgenus Odorrana with phylogenetic reassessment of the related subgenera of the genus Rana. Mol. Phylogenet. Evol. 37, 733-742. 
518 Matsui, T., Matsui, M., 1990. A new brown frog (genus Rana) from Honshu, Japan.

519

520

521

522

523

524

525

526

527

528

529

530

531

532

533

534

535

536

537

538

539

540

541

542

543 Herpetologica 46, 78-85.

Mitsui, Y., Setoguchi, H., 2012. Demographic histories of adaptively diverged riparian and nonriparian species of Ainsliaea (Asteraceae) inferred from coalescent analyses using multiple. BMC Evol. Biol. 12, 254.

Nielsen, R., Wakeley, J., 2001. Distinguishing migration from isolation: a Markov chain Monte Carlo approach. Genetics 158, 885-896.

Pritchard, J., Stephens, M., Donnelly, P., 2000. Inference of population structure using multilocus genotype data. Genetics 155, 945-959.

Rambaut, A., Drummond, A., 2009. Tracer version 1.5. <http://beast.bio.ed.ac.uk/Tracer>.

Reilly, S.B., Marks, S.B., Jennings, W.B., 2012. Defining evolutionary boundaries across parapatric ecomorphs of Black Salamanders (Aneides flavipunctatus) with conservation implications. Mol. Ecol. 21, 5745-5761.

Roelants, K., Gower, D.J., Wilkinson, M., Loader, S.P., Biju, S.D., Guillaume, K., Moriau, L., Bossuyt, F., 2007. Global patterns of diversification in the history of modern amphibians. Proc. Natl. Acad. Sci. U. S. A. 104, 887-892.

Ronquist, F., Huelsenbeck, J.P., 2003. MrBayes 3: Bayesian phylogenetic inference under mixed models. Bioinformatics 19, 1572-1574.

Rovito, S.M., 2010. Lineage divergence and speciation in the Web-toed Salamanders (Plethodontidae: Hydromantes) of the Sierra Nevada, California. Mol. Ecol. 19, 45544571.

Rozas, J., Sanchez-DelBarrio, J.C., Messeguer, X., Rozas, R., 2003. DnaSP, DNA polymorphism analyses by the coalescent and other methods. Bioinformatics 19, 24962497.

Stephens, M., Smith, N., Donnelly, P., 2001. A new statistical method for haplotype reconstruction from population data. Am. J. Hum. Genet. 68, 978-989. 
544 Tajima, F., 1989. Statistical method for testing the neutral mutation hypothesis by DNA $545 \quad$ polymorphism. Genetics 123, 585-595.

546 Tanabe, A.S., 2011. Kakusan4 and Aminosan: two programs for comparing nonpartitioned, 547 proportional and separate models for combined molecular phylogenetic analyses of 548 multilocus sequence data. Mol. Ecol. Resour. 11, 914-921.

549 Tanaka, T., Matsui, M., Takenaka, O., 1996. Phylogenetic relationships of Japanese brown 550 frogs (Rana: Ranidae) assessed by mitochondrial cytochrome b gene sequences. Biochem. $551 \quad$ Syst. Ecol. 24, 299-307.

552 Tanaka-Ueno, T., Matsui, M., Chen, S.-L., Takenaka, O., Ota, H., 1998. Phylogenetic 553 relationships of brown frogs from Taiwan and Japan assessed by mitochondrial $554 \quad$ cytochrome b gene sequences (Rana: Ranidae). Zool. Sci. 15, 283-288.

555 Tavaré, S., 1986. Some probabilistic and statistical problems in the analysis of DNA sequences, in: Miura, R.M. (Ed.), Lectures on Mathematics in the Life Sciences. pp. 57-86.

Waples, R., Gaggiotti, O., 2006. What is a population? An empirical evaluation of some genetic 558 methods for identifying the number of gene pools and their degree of connectivity. Mol. Ecol. 15, 1419-1439.

Woerner, A.E., Cox, M.P., Hammer, M.F., 2007. Recombination-filtered genomic datasets by information maximization. Bioinformatics 23, 1851-1853.

562 Wright, S., 1931. Evolution in Mendelian populations. Genetics 16, 97-159.

563

\section{Supporting information}

Additional supporting information may be found in the online version of this article. 


\section{Table Captions}

568 Table 1 The mean estimated divergence times (MYA) for R. tagoi, R. sakuraii, and the 569 outgroups. Values in parentheses are the 95\% highest posterior density interval. For the node 570 numbers, refer to Fig. 2.

571

572 Table 2 Demographic parameters estimated in the IM analysis. $N_{e}$, effective population size

573 (million individuals); $2 N_{e} M$, effective population migration rate (number of gene

574 copies/generation), for which $2 N_{e} M_{1 \rightarrow 2}\left(2 N_{e} M_{2 \rightarrow 1}\right)$ indicates gene flow from group 1 to 2 (2 to

575 1) forwards in time; $T$, population divergence time (MYA). Values supported by the highest 576 probability are shown as HiPt, and HPD95 indicates the 95\% highest posterior density interval.

577 Parameters in bold indicate the values with statistical support, and characters in italics are those

578 with no significant peak of posterior probability density.

579

$580 \quad$ Figure Captions

581 Fig. 1 Map showing the sampling localities of Rana tagoi tagoi (circles), R. t. yakushimensis

582 (double circle), R. t. okiensis (stars), and R. sakuraii (triangles). Each species lineage inferred 583 using mitochondrial haplotypes is represented by different markers. For the locality information, 584 see Table S1.

585

586 Fig. 2 Maximum-likelihood tree based on the complete mitochondrial $16 S$ and ND1 sequences

587 (2579 bp in total) for Rana tagoi and R. sakuraii. For the locality number, see Fig. 1.

588 Haplotypes in Clades A' and B are sampled from Honshu or the Oki Island (B-1). Haplotypes in 589 Clades A" are from Kyushu, Shikoku, or adjacent small islands.

590

591 Fig. 3 Results of STRUCTURE analyses based on the five nuclear genes. Each species lineage

592 inferred using mitochondrial haplotypes is separated by black vertical lines. (top) The best 
593 clustering result ( $K=2$ clusters) for all 128 samples. (left bottom) Results with $K=2$ (best) and 3

594 for Cluster I. (right bottom) Results with $K=3$ (best) and 4 for Cluster II.

595

596 Fig. 4 Hypothesized scenarios for non-monophyly of mitochondrial haplotypes in $R$. sakuraii:

597 (1) the species-level ILS hypothesis; and (2) the past mitochondrial introgression hypothesis, in

598 which introgression occurred from $R$. tagoi Lineage A-2 to R. sakuraii (a) or in the opposite

599 direction (b). Solid and broken lines indicate the mitochondrial lineages of $R$. tagoi and $R$.

600 sakuraii, respectively. Grey arrows indicate massive mitochondrial introgression.

601

602 Captions for supplementary materials

603 Table S1 The samples used in this study with information on the sampling localities, vouchers,

604 and GenBank accession numbers for each locus. KUHE, Graduate School of Human and

605 Environmental Studies, Kyoto University; TMP, temporary number.

606

607

Table S2 The primers used to amplify mt- and n-DNA in this study.

608

609

Table S3 Summary statistics of each locus. Tajima's $D$ values; length of sequence after

610 alignment; variable sites (vs); number of haplotypes $(h)$; haplotype diversity $(H d)$; and

611 nucleotide diversity $(\pi)$.

612

613 Fig. S1 Median-joining networks of five nuclear loci. The size of each circle reflects the relative 614 sample size of each haplotype. The color indicates nuclear clusters and species as follows: red = 615 n-Cluster I of $R$. tagoi; green = n-Cluster II of $R$. tagoi; light green = n-Cluster II of $R$. sakuraii. 616 Black circles and bars indicate median vectors and missing haplotypes, respectively. 
618 Fig. S2 Posterior probability densities for divergence time (T), effective population size $\left(N_{e}\right)$,

619 and population migration rate $\left(2 \mathrm{NeM}_{e}\right)$ of Clusters I and II obtained in the IM analyses. The

620 resultant values and 95\% confidence intervals for each estimate are listed in Table 2.

621

622 Fig. S3 Posterior probability densities for divergence time ( $T$, left top), effective population size

623 ( $N_{e}$, left middle and bottom), and population migration rate (2NeM, right) of $R$. tagoi (Rt)

624 lineage A-2, A-5+6, and R. sakuraii (Rs). Estimates with no statistical support are indicated by

625 ns. The parameters obtained in three- and two-populations models are shown as triangles and

626 circles, respectively. The resultant values and 95\% confidence intervals for each estimate are

627 listed in Table 2.

628

629

630 The English in this document has been checked by at least two professional editors, both native

631 speakers of English. For a certificate, please see:

632 http://www.textcheck.com/

633 certificate/wc1m0N

634 
Table 1 The mean estimated divergence times

(MYA) for $R$. tagoi , $R$. sakuraii , and the outgroups. Values in parentheses are the 95\% highest posterior density interval. For the node numbers, refer to Fig.

\begin{tabular}{ccc}
\hline Node & Calibration I & Calibration II \\
\hline 1 & $4.00(5.96-2.33)$ & $4.16(6.16-2.44)$ \\
2 & $2.58(3.82-1.60)$ & $2.82(4.07-1.69)$ \\
3 & $2.31(3.38-1.42)$ & $2.46(3.54-1.45)$ \\
4 & $1.84(2.69-1.05)$ & $1.73(2.60-0.99)$ \\
5 & $1.16(1.87-0.60)$ & $1.04(1.66-0.50)$ \\
6 & $1.32(2.00-0.69)$ & $1.35(2.06-0.70)$ \\
7 & $1.87(2.78-1.05)$ & $2.08(3.07-1.21)$ \\
8 & $0.95(1.60-0.44)$ & $1.13(1.78-0.58)$ \\
9 & $0.88(1.40-0.41)$ & $1.39(2.16-0.72)$ \\
10 & $1.92(2.85-1.09)$ & $2.12(3.14-1.24)$ \\
11 & $1.75(2.59-0.96)$ & $1.69(2.50-0.90)$ \\
12 & $0.50(0.95-0.14)$ & $0.42(0.81-0.11)$ \\
13 & $1.15(1.85-0.56)$ & $1.50(2.30-0.82)$ \\
14 & $0.36(0.64-0.13)$ & $0.53(0.90-0.21)$ \\
15 & $2.31(3.36-1.34)$ & $2.54(3.70-1.53)$ \\
16 & $0.79(1.30-0.34)$ & $0.85(1.38-0.37)$ \\
17 & $0.20(0.43-0.03)$ & $0.17(0.37-0.01)$ \\
18 & $1.68(2.53-0.99)$ & $1.98(2.91-1.19)$ \\
19 & $0.53(0.90-0.21)$ & $0.59(1.01-0.24)$ \\
20 & $1.04(1.67-0.46)$ & $1.40(2.11-0.73)$ \\
21 & $1.46(2.22-0.76)$ & $1.54(2.34-0.83)$ \\
22 & $2.71(4.34-1.40)$ & $2.29(3.50-1.24)$ \\
23 & $1.40(2.15-0.71)$ & $1.35(2.07-0.71)$ \\
24 & $0.80(1.28-0.36)$ & $0.82(1.32-0.39)$ \\
25 & $0.90(1.46-0.42)$ & $1.08(1.74-0.52)$ \\
26 & $0.04(0.13-0.03)$ & $0.26(0.53-0.05)$ \\
O-1 & $22.02(35.97-11.08)$ & $11.59(18.29-6.42)$ \\
\hline & &
\end{tabular}


'I'able 2 Demographic parameters estimated in the IM analysis. $N_{e}$, ettective population size (million individuals); $2 N_{e} M$, effective population migration rate (number of gene copies/generation), for which $2 N_{e} M_{1 \rightarrow 2}\left(2 N_{e} M_{2 \rightarrow 1}\right)$ indicates gene flow from group 1 to 2 (2 to 1 ) forwards in time; $T$, population divergence time (MYA). Values supported by the highest probability are shown as HiPt, and HPD95 indicates the 95\% highest posterior density interval. Parameters in bold indicate the values with statistical support and characters in italics are those with no significant peak of posterior nrobabilitv densitv

\begin{tabular}{|c|c|c|c|c|c|c|}
\hline & $N_{1}$ & $N_{2}$ & $N_{\text {ancestor }}$ & $2 N_{e} M_{1 \rightarrow 2}$ & $2 N_{e} M_{2 \rightarrow 1}$ & $T$ \\
\hline \multicolumn{7}{|c|}{ (1) Cluster I vs. (2) Cluster II } \\
\hline $\begin{array}{l}\text { HiPt } \\
\text { HPD95 }\end{array}$ & $\begin{array}{c}2.18 \\
(1.70-2.87)\end{array}$ & $\begin{array}{c}1.73 \\
(1.34-2.30)\end{array}$ & $\begin{array}{c}0.40 \\
(0.17-0.79)\end{array}$ & $\begin{array}{c}\mathbf{0 . 5 2} \\
(0.24-1.12)\end{array}$ & $\begin{array}{c}1.23 \\
(0.70-2.14)\end{array}$ & $\begin{array}{c}2.72 \\
(2.10-4.29)\end{array}$ \\
\hline
\end{tabular}

Three-pops. model: (1) R. sakuraii vs. (2) R. tagoi lin
HiPt
0.16
0.80
0.21
0.01
0.00
1.05
HPD95
$(0.07-0.32)$
$(0.34-2.06)$
$(0.01-4.06)$
$(0.00-2.46)$
$(0.00-0.84)$
$(0.63-2.26)$

Three-pops. model: (1) R. sakuraii vs. (2) R. tagoi lineage A-5, 6
HiPt
0.16
0.79
0.46
0.40
HPD95
$(0.07-0.31)$
$(0.38-1.76)$
$(0.00-2.52)$
$(0.04-2.00)$

Three-pops. model: (1) R. tagoi A-2 vs. (2) R. tagoi A-5, 6
HiPt
0.80
0.79
0.17
HPD95
$(0.34-2.06)$
$(0.38-1.76)$
$(0.00-3.74)$
3.79
$(0.75-9.50)$

Three-pops. model: (1) ancestor of $R$. sakuraii and R. tagoi A-2 vs. (2) R. tagoi A-5, 6
$\mathrm{HiPt}$
0.21
0.79
0.43
0.06
0.04
2.15
HPD95
(0.01-4.06)
$(0.38-1.76)$
$(0.23-0.77)$
$(0.00-81.15)(0.00-34.07)$
$(1.31-6.11)$

Two-pops. model: (1) $R$. sakuraii vs. (2) R. tagoi A-2, 5, 6
HiPt
0.17
1.61
0.37
0.01
0.51
1.21
HPD95
$(0.09-0.34) \quad(0.99-2.65)$
$(0.10-0.68) \quad(0.00-3.19)$
$(0.14-1.17)$
$(0.56-2.85)$ 
Table S1 The samples used in this study with information on the sampling localities, vouchers, and GenBank accession numbers for each locus. KUHE, Graduate School of Human and Environmental Studies, Kyoto University; TMP, temporary number.

\begin{tabular}{|c|c|c|c|c|c|c|c|c|}
\hline \multirow{2}{*}{$\begin{array}{l}\text { loc. locality } \\
\text { nos. }\end{array}$} & \multirow{2}{*}{$\begin{array}{l}\text { voucher } \\
\text { (KUHE) }\end{array}$} & \multirow{2}{*}{ mt-lineage } & \multicolumn{6}{|c|}{ GenBank accession no. } \\
\hline & & & $\operatorname{mtDNA}(16 S, N D 1)$ & $N C X 1$ & NFIA & POMC & SLC8A3 & TYR \\
\hline
\end{tabular}

\section{Rana tagoi tagoi}

1 Mutsu City, Aomori Pref.

2 Noshiro City, Akita Pref.

3 Ichinoseki City, Iwate Pref.

4 Sendai City, Miyagi Pref.

5 Yamagata City, Yanagata Pref.

6 Nihonmatsu City, Fukushima Pref.

7 Nihonmatsu City, Fukushima Pref.

8 Daigo town, Ibaraki Pref.

9 Tsukuba City, Ibaraki Pref.

10 Ichihara City, Chiba Pref.

11 Kanuma City, Tochigi Pref.

12 Uonuma City, Nigata Pref.

13 Nakanojo Town, Gunma Pref.

14 Saku City, Nagano Pref.

15 Akiruno City, Tokyo Pref.

16 Fujikawaguchiko Town, Yamanashi Pref.

17 Minobu Town, Yamanashi Pref.

18 Izu City, Shizuoka Pref.

19 Hokuto City, Yamanashi Pref.

23 Nagano City, Nagano Pref.

24 Kurobe City, Toyama Pref.

\section{Takayama City, Gifu Pref.}

26 Gujo City, Gifu Pref.

27 Fujieda City, Shizuoka Pref.

28 Neba Village, Nagano Pref.

29 Shinjo City, Aichi Pref.

30 Okazaki City, Aichi Pref.

31 Ise City, Mie Pref.

33 Ibigawa Town, Gifu Pref.

34 Takashima City, Shiga Pref.

35 Taga Town, Shiga Pref.

36 Matsuzaka City, Mie Pref.

37 Joyo City, Kyoto Pref.

38 Odai Town, Mie Pref.

39 Gobo City, Wakayama Pref.

40 Kyoto City, Kyoto Pref.

41 Nantan City, Kyoto Pref.

42 Sasayama City, Hyogo Pref.

43 Kobe City, Hyogo Pref.

44 Taka Town, Hyogo Pref.

45 Kyotango City, Kyoto Pref.

46 Toyooka City, Hyogo Pref.

47 Wakasa Town, Tottori Pref.

49 Mimasaka City, Okayama Pref.

52 Misasa Town, Tottori Pref.

53 Kagamino Town, Okayama Pref.

54 Shobara City, Hiroshima Pref.

55 Izumo City, Shimane Pref.

57 Higashihiroshima City, Hiroshima Pref.

58 Hatsukaichi City, Hiroshima Pref.

60 Shimonoseki City, Yamaguchi Pref.

61 Minamiawaji City, Hyogo Pref.

62 Manno Town, Kagawa Pref.

63 Miyoshi City, Tokushima Pref.
44827

46598

36699

45622

37543

29595

36330

42344

43723

43886

TMP_081122-1

42747

28409

46172

40166

36896

44810

44811

22930

22936

44797

43980

42452

42453

45558

43480

45552

45549

43468

43483

18005

45102

45103

45014

45099

42048

43018

14228

17955

27335

27337

45913

45910

42829

27388

43925

43924

43512

41484

41554

40190

45047

41229

42342

44828

42319

41408

41405

41430

10307

45392

10330

14171

42711

42714

34743

27659

24574

29739

36040

18877

30262

unnumbered

43167

34516

43885

TMP_T2882

TMP_T3498
A-1a

A-1a

A-1a

A-1a

A-1a

A-2

A-1a

A-1a

A-2

A-2

A-2

A-2

A-2

A-1a

A-1a

A-1a

A-1a

A-4

A-4

A-4

A-2

A-2

A-2

A-2

A-6

A-2

A-6

A-2

A-5

A-5

A-1a

A-1a

A-5

A-5

A-1a

A-1a

A-5

A-6

A-6

A-6

A-6

A-6

A-6

A-1a

A-1a

A-1b

B-2a

B-2a

B-2a

B-2a

B-2a

B-2a

A-1b

A-1b

B-2a

A-1b

B-2a

B-2a

A-1b

A-1b

B-2a

A-1b

A-1b

B-2a

A-1b

B-2a

B-2b

$\mathrm{B}-2 \mathrm{~b}$

B-2b

B-2b

$\mathrm{B}-2 \mathrm{~b}$

B-2b

B-2b

B-2b

A-7

A-7

A-7

AB639413, AB639593 AB968741 AB968871 AB968996 AB969125 AB969253 AB968306 AB968765 AB968894 AB969021 AB969150 AB969279 AB639413, AB639598 AB968687 AB968816 AB968942 AB969071 AB969199 AB968302 AB968761 AB968890 AB969017 AB969146 AB969274 AB639417, AB639601 AB968689 AB968818 AB968944 AB969073 AB969201 AB639419, AB639604 AB968676 AB968805 AB968931 AB969060 AB969189 AB639474, AB639643 AB968686 AB968815 AB968941 AB969070 AB969198 AB639420, AB639605 AB968703 AB968832 AB968958 AB969087 AB969215 AB968270 AB968725 AB968854 AB968980 AB969109 AB969237

AB639421, AB639646 AB968728 AB968857 AB968983 AB969112 AB969240 AB968251 AB968772 AB968784 AB969029 AB969156 AB969158 AB639479, AB639648 AB968709 AB968838 AB968964 AB969093 AB969221 AB639482, AB639652 AB968673 AB968803 AB968929 AB969058 AB969186 AB968305 AB968764 AB968893 AB969020 AB969149 AB969277 AB639422, AB639609 AB968690 AB968819 AB968945 AB969074 AB969202 AB639429, AB639612 AB968688 AB968817 AB968943 AB969072 AB969200 AB968281 AB968739 AB968869 AB968994 AB969123 AB969251 AB968282 AB968740 AB968870 AB968995 AB969124 AB969252 AB639487, AB639657 AB968658 AB968787 AB968913 AB969042 AB969171 AB639487, AB639657 AB968659 AB968788 AB968914 AB969043 AB969172 AB968280 AB968738 AB968868 AB968993 AB969122 AB969250 AB968274 AB968732 AB968861 AB968987 AB969116 AB969244 AB639483, AB639651 AB968705 AB968834 AB968960 AB969089 AB969217 AB968263 AB968706 AB968835 AB968961 AB969090 AB969218 AB968300 AB968759 AB968888 AB969015 AB969144 AB969272

AB639493, AB639663 AB968716 AB968845 AB968971 AB969100 AB969228 AB968299 AB968758 AB968887 AB969014 AB969143 AB969271 AB968298 AB968757 AB968886 AB969013 AB969142 AB969270 AB639485, AB639655 AB968715 AB968844 AB968970 AB969099 AB969227 AB639489, AB639659 AB968717 AB968846 AB968972 AB969101 AB969229 AB639488, AB639658 AB968654 AB968782 AB968909 AB969038 AB969167 AB968287 AB968746 AB968876 AB969002 AB969131 AB969259 AB968288 AB968747 AB968877 AB969003 AB969132 AB969260 AB968283 AB968742 AB968872 AB968998 AB969127 AB969255 AB968286 AB968745 AB968875 AB969001 AB969130 AB969258 AB968261 AB968700 AB968829 AB968955 AB969084 AB969212 AB639434, AB639617 AB968711 AB968840 AB968966 AB969095 AB969223 AB639490, AB639660 AB968652 AB968780 AB969027 AB969036 AB969165 AB639498, AB639668 AB968653 AB968781 AB968908 AB969037 AB969166 AB639500, AB639670 AB968665 AB968794 AB968920 AB969049 AB969178 AB968254 AB968666 AB968795 AB968921 AB969050 AB969179 AB968304 AB968763 AB968892 AB969019 AB969148 AB969276 AB968303 AB968762 AB968891 AB969018 AB969147 AB969275 AB639502, AB639672 AB968710 AB968839 AB968965 AB969094 AB969222 AB639436, AB639619 AB968667 AB968796 AB968922 AB969051 AB969180 AB968273 AB968731 AB968860 AB968986 AB969115 AB969243 AB968272 AB968730 AB968859 AB968985 AB969114 AB969242 AB968266 AB968718 AB968847 AB968973 AB969102 AB969230 AB639551, AB639716 AB968698 AB968827 AB968953 AB969082 AB969210 AB639549, AB639714 AB968699 AB968828 AB968954 AB969083 AB969211 AB639553, AB639718 AB968691 AB968820 AB968946 AB969075 AB969203 AB968284 AB968743 AB968873 AB968999 AB969128 AB969257 AB639561, AB639727 AB968693 AB968822 AB968948 AB969077 AB969205 AB968262 AB968702 AB968831 AB968957 AB969086 AB969214 AB968307 AB968768 AB968896 AB968997 AB969126 AB969254 AB639464, AB639712 AB968701 AB968830 AB968956 AB969085 AB969213 AB639452, AB639630 AB968695 AB968824 AB968950 AB969079 AB969207 AB968259 AB968694 AB968823 AB968949 AB969078 AB969206 AB968260 AB968697 AB968826 AB968952 AB969081 AB969209 AB639469, AB639639 AB968647 AB968776 AB968903 AB969031 AB969160 AB968297 AB968756 AB968885 AB969012 AB969141 AB969269 AB639564, AB639729 AB968648 AB968777 AB968904 AB969032 AB969161 $\begin{array}{lllll}\text { AB968253 AB968651 AB968779 AB968907 AB969035 AB969164 } & \text { AB }\end{array}$ AB639466, AB639637 AB968707 AB968836 AB968962 AB969091 AB969219 AB639467, AB639729 AB968708 AB968837 AB968963 AB969092 AB969220 AB639473, AB639642 AB968684 AB968813 AB968939 AB969068 AB969196 AB639464, AB639730 AB968670 AB968800 AB968926 AB969055 AB969183 AB639465, AB639731 AB968660 AB968789 AB968915 AB969044 AB969173 AB968256 AB968677 AB968806 AB968932 AB969061 AB969190 AB639469, AB639734 AB968685 AB968814 AB968940 AB969069 AB969197 AB639467, AB639734 AB968655 AB968783 AB968910 AB969039 AB969168 AB639472, AB639737 AB968678 AB968807 AB968933 AB969062 AB969191 AB639571, AB639736 AB968773 AB968901 AB969028 AB969157 AB969281 AB968265 AB968713 AB968842 AB968968 AB969097 AB969225 
64 Toyo Town, Kochi Pref.

65 Saijo City, Ehime Pref.

66 Saiyo City, Ehime Pref.

67 Kitakyushu City, Fukuoka Pref.

68 Beppu City, Oita Pref.

69 Yatsushiro City, Kumamoto Pref.

70 Amakusa City, Kumamoto Pref.

71 Kanoya City, Kagishima Pref.

72 Sasebo City, Nagasaki Pref.

73 Goto City, Nagasaki Pref.

74 Nobeoka City, Miyazaki Pref.

75 Nishimera Village, Miyazaki Pref.

76 Miyakonojo City, Miyazaki Pref.

77 Kimotsuki City, Kagoshima Pref.

78 Kinko Town, Kagoshima Pref.

79 Goto City, Nagasaki Pref.

79 Shinkamigoto City, Nagasaki Pref.

80 Goto City, Nagasaki Pref.

R. t. okiensis

50 Okinoshima Town, Shimane Pref.

51 Nishinoshima Town, Shimane Pref R. t. yakushimensis

81 Yakushima Town, Kagoshima Pref.

R. sakuraii

11 Kanuma City, Tochigi Pref.

15 Akiruno City, Tokyo Pref.

17 Minobu Town, Yamanashi Pref.

20 Shizuoka City, Shizuoka Pref.

21 Shizuoka City, Shizuoka Pref.

22 Matsumoto City, Nagano Pref.

24 Kurobe City, Toyama Pref.

24 Kurobe City, Toyama Pref.

32 Katsuyama City, Fukui Pref.

38 Odai Town, Mie Pref.

41 Nantan City, Kyoto Pref.

48 Wakasa Town, Tottori Pref.

59 Iwakuni City, Yamaguchi Pref.

R. tsushimensis R. kobai

Tsushima City, Nagasaku Pref.

Amami City, Kagoshima Pref.

R. ulma

Higashi Village, Okinawa Pref.

$R$. sauteri

Chiayi County, Taiwan
29464

27679

43078

TMP_T2241

28612

43637

27562

30342

27295

27140

45359

45362

27121

26088

30907

43397

27678

31539

45149

TMP_110216-1

44316

44317

45355

10818

22341

43647

10182

45177

45182

43633

43634

43635

42450

43740

45620

unnumbered

44254

44286

44256

22887

45105

45106

43591

27647

40309

45049

41412

unnumbered

34740

43893

10606

10051

10056
A-7

A-7

A-7

A-7

A-9a

A-9a

A-9a

A-9a

A-9a

A-9a

A-9a

A-9a

A-9b

A-9b

A-9b

A-9b

A-9b

A-9c

A-9C

A-9c

A-9c

A-9C

A-9c

B-1

B-1

B-1

A-8

A-8

A-8

A-2

A-2

A-2

A-2

A-2

A-2

A-2

A-3

A-3

A-2

A-2

A-3

A-3

A-3

A-3

A-3

A-3

A-3

A-3

A-3

A-3

6894

AB639510, AB639679 AB968675 AB968804 AB968930 AB969059 AB969188 AB639507, AB639676 AB968672 AB968802 AB968928 AB969057 AB969185 AB968264 AB968712 AB968841 AB968967 AB969096 AB969224 AB639509, AB639678 AB968769 AB968897 AB969023 AB969152 AB969282 AB968255 AB968674 AB968798 AB968924 AB969053 AB969187 AB639519, AB639688 AB968723 AB968852 AB968978 AB969107 AB969235 AB639524, AB639691 AB968668 AB968797 AB968923 AB969052 AB969181 AB639525, AB639692 AB968679 AB968808 AB968934 AB969063 AB969192 AB639530, AB639697 AB968664 AB968793 AB968919 AB969048 AB969177 AB639518, AB639687 AB968663 AB968792 AB968918 AB969047 AB969176 $\begin{array}{llllll}\text { AB968295 } & \text { AB968754 AB968883 AB969010 AB969139 AB969267 }\end{array}$ AB968296 AB968755 AB968884 AB969011 AB969140 AB969268 AB639528, AB639695 AB968662 AB968791 AB968917 AB969046 AB969175 AB639529, AB639696 AB968661 AB968790 AB968916 AB969045 AB969174 AB639532, AB639699 AB968680 AB968809 AB968935 AB969064 AB969193 AB639533, AB639700 AB968714 AB968843 AB968969 AB969098 AB969226 AB639536, AB639703 AB968671 AB968801 AB968927 AB969056 AB969184 AB639538, AB639705 AB968681 AB968810 AB968936 AB969065 AB969194 AB968291 AB968750 AB968879 AB969006 AB969135 AB969263 AB968252 AB968650 AB968774 AB968906 AB969034 AB969163 AB968278 AB968736 AB968866 AB968991 AB969120 AB969248 AB968279 AB968737 AB968867 AB968992 AB969121 AB969249 AB968294 AB968753 AB968882 AB969009 AB969138 AB969266

AB639576, AB639742 AB968649 AB968778 AB968905 AB969033 AB969162 AB639579, AB639742 AB968656 AB968785 AB968911 AB969040 AB969169 AB639580, AB639742 AB968724 AB968853 AB968979 AB969108 AB969236

AB639578, AB639741 AB968646 AB968775 AB968902 AB969030 AB969159 AB968292 AB968751 AB968880 AB969007 AB969136 AB969264 AB968293 AB968752 AB968881 AB969008 AB969137 AB969265

AB968268 AB968720 AB968849 AB968975 AB969104 AB969232 AB968269 AB968721 AB968850 AB968976 AB969105 AB969233 AB639423, AB639744 AB968722 AB968851 AB968977 AB969106 AB969234 AB639583, AB639744 AB968704 AB968833 AB968959 AB969088 AB969216 AB968271 AB968726 AB968855 AB968981 AB969110 AB969238 AB968301 AB968760 AB968889 AB969016 AB969145 AB969273

AB639488, AB639749 AB968766 AB968900 AB969022 AB969151 AB969280 AB968275 AB968733 AB968862 AB968988 AB969117 AB969246 AB968277 AB968735 AB968865 AB968990 AB969119 AB969247 AB968276 AB968734 AB968863 AB968989 AB969118 AB969245 AB639585, AB639746 AB968657 AB968786 AB968912 AB969041 AB969170 AB968289 AB968748 AB968878 AB969004 AB969133 AB969261 AB968290 AB968749 AB968864 AB969005 AB969134 AB969262 AB968267 AB968719 AB968848 AB968974 AB969103 AB969231 AB639554, AB639719 AB968669 AB968799 AB968925 AB969054 AB969182 AB639555, AB639720 AB968692 AB968821 AB968947 AB969076 AB969204 AB968285 AB968744 AB968874 AB969000 AB969129 AB969256 AB639455, AB639632 AB968696 AB968825 AB968951 AB969080 AB969208 AB639454, AB639631 AB968767 AB968895 AB969026 AB969155 AB969285 AB968257 AB968683 AB968812 AB968938 AB969067 AB969195 AB639590, AB639750 AB968729 AB968858 AB968984 AB969113 AB969241

AB639592, AB639752

AB685768

AB685780

AB685767 
Table S2 The primers used to amplify mt- and n-DNA in this study.

\begin{tabular}{|c|c|c|c|}
\hline Target & Name & Sequence & Reference \\
\hline \multirow[t]{8}{*}{165} & L1507 & TACACACCGCCCGTCACCCTCTT & Shimada et al (2011) \\
\hline & H1923 & AAGTAGCTCGCTTAGTTTCGG & Shimada et al (2011) \\
\hline & L1879 & CGTACСТTTTGCATCATGGTC & Shimada et al (2011) \\
\hline & H2315 & TTCTTGTTACTAGTTCTAGCAT & Shimada et al (2011) \\
\hline & L2188 & AAAGTGGGCCTAAAAGCAGCCA & Matsui et al (2006) \\
\hline & Wilkinson_t & 6 CCCTCGTGATGCCGTTGATAC & Wilkinson et al (2002 \\
\hline & $16 \mathrm{~L} 1$ & CTGACCGTGCAAAGGTAGCGTAATCACT & Hedges (1994) \\
\hline & $16 \mathrm{H} 1$ & CTCCGGTCTGAACTCAGATCACGTAGG & Hedges (1994) \\
\hline \multirow[t]{4}{*}{ ND1 } & L3032 & CGACCTCGATGTTGGATCAGG & Shimada et al (2011) \\
\hline & ND1_Htago & GRGCRTATTTGGAGTTTGARGCTC & \\
\hline & ND1_Ltag & GACCTAAACCTCAGYATYCTATTTAT & Eto et al (2012) \\
\hline & tMet_H & AGGAAGTACAAAGGGTTTTGATC & Shimada et al (2011) \\
\hline \multirow[t]{2}{*}{ NCX1 } & NCX1F & ACAACAGTRAGRATATGGAA & Shimada et al. (2011) \\
\hline & NCX1R1 & GCCATATCTCTCCTCGCTTCTTC & Eto et al (2013) \\
\hline \multirow[t]{2}{*}{ NFIA } & NFIA-005_1 & FTTTGTCACATCAGGTGTTTT & This study \\
\hline & NFIA-005_I & ECTTGCCTTGGCTGCT & \\
\hline \multirow[t]{2}{*}{ POMC } & POMC1 & GAATGTATYAAAGMMTGCAAGATGGW & CWiens et al. (2005) \\
\hline & POMC7 & TGGCATTTTTGAAAAGAGTCAT & Smith et al. (2005) \\
\hline \multirow[t]{2}{*}{ SLC8A3 } & SCF_2F & CAAACACAGRGSAATTATGAT & Shimada et al (2011) \\
\hline & SCF_2R & ATAATYCCAACTGARAACTC & Shimada et al (2011) \\
\hline \multirow[t]{2}{*}{ TYR } & Tyr_L1 & CCCCAGTGGGYRCCCARTTCCC & Kuraishi et al (2013) \\
\hline & & CCACCTTCTGGATTTCCCGTTC & Kuraishi et al (2013) \\
\hline
\end{tabular}

\section{References}

Eto, K., Matsui, M., Sugahara, T., 2013. Discordance between mitochondrial DNA genealogy and nuclear DNA genetic structure in the two morphotypes of Rana tagoi tagoi (Amphibia: Anura: Ranidae) in the Kinki Region, Japan. Zool. Sci. 30, 553-558. Eto, K., Matsui, M., Sugahara, T., Tanaka-Ueno, T., 2012. Highly complex mitochondrial DNA genealogy in an endemic Japanese subterranean breeding brown frogRana tagoi (Amphibia, Anura, Ranidae). Zool. Sci. 29, 662-671.

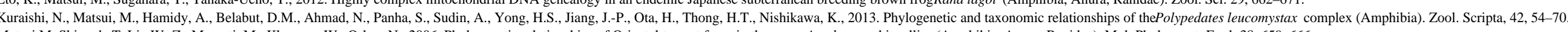
Matsui M, Shimada T, Liu W-Z., Maryati, M., Khonsue, W., Orlov, N., 2006. Phylogenetic relationships of Oriental torrent frogs in the genus Amolops and its allies (Amphibia, Anura, Ranidae). Mol. Phylogenet. Evol. 38, 659-666. Shimada, T., Matsui, M., Yambun, P., Sudin, A. 2011. A taxonomic study of Whitehead's torrent frog, Meristogenys whiteheadi, with descriptions of two new species (Amphibia: Ranidae). Zool. J. Linnean, Soc, 161, 157-183.

Smith, S.A., Stephens, P.R., Wiens, J., 2005. Replicate patterns of species richness, historical biogeography, and phylogeny in Holarctic treefrogs. Evolution, 59, 2433-2450.

Wiens, J., Fetzner, J.W. Jr, Parkinson, C.L., Reeder, T.W., 2005. Hylid frog phylogeny and sampling strategies for species clades. Syst. Biol. 54, 719-748,

Wilkinson, J.A., Drewes, R.C., Tatum, O.L., 2002. A molecular phylogenetic analysis of the family Rhacophoridae with an emphasis on the Asian and African genera. Mol. Phylogenet. Evol. 24, 265-273. 
Table S3 Summary statistics of each locus. Tajima's $D$ values; length of sequence after alignment; variable sites (vs); number of haplotypes ( $h$ ); haplotype diversity $(H d)$; and nucleotide diversity $(\pi)$.

\begin{tabular}{|c|c|c|c|c|c|c|}
\hline & ajima's & ite & VS & $h$ & $H d$ & $\pi$ \\
\hline & \multicolumn{6}{|c|}{ whole $(\mathrm{n}=128)$} \\
\hline $16 S$ & -1.307 & 1612 & 285 & 115 & 0.998 & 0.024 \\
\hline ND1 & -0.816 & 967 & 287 & 104 & 0.997 & 0.047 \\
\hline NCX1 (SLC8A1) & -0.648 & 505 & 26 & 37 & 0.851 & 0.006 \\
\hline$N F I A$ & -1.626 & 414 & 18 & 21 & 0.735 & 0.003 \\
\hline POMC & -1.538 & 475 & 40 & 48 & 0.870 & 0.007 \\
\hline SLC8A3 (NCX3 & -1.429 & 524 & 21 & 23 & 0.786 & 0.003 \\
\hline TYR & -1.210 & 318 & 50 & 97 & 0.955 & 0.017 \\
\hline
\end{tabular}

\begin{tabular}{|c|c|c|c|}
\hline VS & $h$ & $H d$ & $\pi$ \\
\hline \multicolumn{4}{|c|}{ mt-lineage A-1a $(\mathrm{n}=18)$} \\
\hline 44 & 15 & 0.980 & 0.006 \\
\hline 44 & 14 & 0.974 & 0.010 \\
\hline 7 & 5 & 0.651 & 0.003 \\
\hline 3 & 5 & 0.548 & 0.002 \\
\hline 9 & 6 & 0.712 & 0.004 \\
\hline 3 & 4 & 0.236 & 0.001 \\
\hline 17 & 12 & 0.867 & 0.013 \\
\hline
\end{tabular}

\begin{tabular}{cccc}
$v s$ & $h$ & $H d$ & $\pi$ \\
\hline \multicolumn{4}{c}{ mt-lineage A-1b $(\mathrm{n}=9)$} \\
\hline 50 & 9 & 1.000 & 0.010 \\
70 & 9 & 1.000 & 0.026 \\
3 & 4 & 0.525 & 0.002 \\
3 & 4 & 0.700 & 0.003 \\
7 & 6 & 0.775 & 0.004 \\
2 & 3 & 0.242 & 0.000 \\
15 & 12 & 0.958 & 0.018 \\
\hline
\end{tabular}

\begin{tabular}{|c|c|c|c|}
\hline vS & $h$ & $H d$ & $\pi$ \\
\hline \multicolumn{4}{|c|}{ mt-lineage A-2Rt $(\mathrm{n}=12)$} \\
\hline 51 & 20 & 0.970 & 0.010 \\
\hline 53 & 10 & 0.970 & 0.016 \\
\hline 5 & 4 & 0.649 & 0.003 \\
\hline 2 & 3 & 0.177 & 0.000 \\
\hline 12 & 10 & 0.859 & 0.006 \\
\hline 8 & 6 & 0.659 & 0.002 \\
\hline 20 & 15 & 0.946 & 0.018 \\
\hline
\end{tabular}

$16 S$

ND1

NCX1 (SLC8A1)

NFIA

POMC

SLC8A3 (NCX3)

TYR

\begin{tabular}{|c|c|c|c|}
\hline \multicolumn{4}{|c|}{ mt-lineage A-2Rs $(\mathrm{n}=9)$} \\
\hline 26 & 7 & 0.964 & 0.006 \\
\hline 38 & 7 & 0.964 & 0.016 \\
\hline 2 & 2 & 0.125 & 0.001 \\
\hline 1 & 2 & 0.125 & 0.000 \\
\hline 6 & 5 & 0.556 & 0.003 \\
\hline 1 & 2 & 0.125 & 0.000 \\
\hline 11 & 4 & 0.442 & 0.008 \\
\hline
\end{tabular}

\begin{tabular}{cccc}
\hline \multicolumn{4}{c}{ mt-lineage A-3 $(\mathrm{n}=12)$} \\
\hline 43 & 12 & 1.000 & 0.009 \\
37 & 8 & 0.939 & 0.013 \\
2 & 3 & 0.163 & 0.000 \\
2 & 3 & 0.163 & 0.000 \\
9 & 5 & 0.652 & 0.004 \\
2 & 3 & 0.554 & 0.001 \\
9 & 5 & 0.493 & 0.007 \\
\hline
\end{tabular}

\begin{tabular}{cccc}
\hline \multicolumn{4}{c}{ mt-lineage A-4 $(\mathrm{n}=3)$} \\
\hline 7 & 2 & 0.667 & 0.003 \\
9 & 2 & 0.667 & 0.006 \\
3 & 2 & 0.533 & 0.003 \\
2 & 3 & 0.600 & 0.002 \\
3 & 2 & 0.533 & 0.003 \\
1 & 2 & 0.533 & 0.001 \\
1 & 2 & 0.533 & 0.002 \\
\hline
\end{tabular}

\begin{tabular}{cccc}
\hline \multicolumn{4}{c}{ mt-lineage A-5 $(\mathrm{n}=5)$} \\
\hline 41 & 5 & 1.000 & 0.012 \\
38 & 4 & 0.900 & 0.018 \\
3 & 3 & 0.607 & 0.002 \\
1 & 2 & 0.250 & 0.001 \\
4 & 3 & 0.607 & 0.003 \\
1 & 2 & 0.571 & 0.001 \\
12 & 7 & 0.964 & 0.018 \\
\hline
\end{tabular}

16S

\begin{tabular}{cccc}
\hline \multicolumn{4}{c}{ mt-lineage A-6 $(\mathrm{n}=8)$} \\
\hline 25 & 8 & 1.000 & 0.005 \\
15 & 6 & 0.964 & 0.005 \\
4 & 5 & 0.505 & 0.002 \\
2 & 3 & 0.425 & 0.001 \\
9 & 7 & 0.850 & 0.006 \\
2 & 2 & 0.363 & 0.001 \\
16 & 13 & 0.975 & 0.016 \\
\hline
\end{tabular}

\begin{tabular}{cccc}
\hline \multicolumn{4}{c}{ mt-lineage A-7 $(\mathrm{n}=7)$} \\
\hline 27 & 7 & 1.000 & 0.006 \\
32 & 7 & 1.000 & 0.011 \\
2 & 3 & 0.538 & 0.001 \\
2 & 3 & 0.275 & 0.001 \\
- & 1 & - & - \\
2 & 2 & 0.440 & 0.002 \\
9 & 7 & 0.846 & 0.009 \\
\hline
\end{tabular}

\begin{tabular}{cccc}
\hline \multicolumn{4}{c}{ mt-lineage A-8 $(\mathrm{n}=3)$} \\
\hline 3 & 2 & 0.667 & 0.001 \\
3 & 2 & 0.667 & 0.002 \\
4 & 3 & 0.733 & 0.004 \\
2 & 3 & 0.600 & 0.002 \\
4 & 3 & 0.600 & 0.003 \\
- & 1 & - & - \\
10 & 4 & 0.800 & 0.013 \\
\hline
\end{tabular}

\begin{tabular}{cccc}
\hline \multicolumn{4}{c}{ mt-lineage A-9a $(\mathrm{n}=8)$} \\
\hline 28 & 7 & 0.964 & 0.006 \\
21 & 7 & 0.964 & 0.006 \\
6 & 6 & 0.792 & 0.004 \\
2 & 3 & 0.433 & 0.001 \\
2 & 3 & 0.242 & 0.001 \\
1 & 2 & 0.264 & 0.001 \\
11 & 7 & 0.692 & 0.009 \\
\hline
\end{tabular}

16S

ND1
NCX1 (SLC8A1)
NFIA

\begin{tabular}{cccc}
\hline \multicolumn{4}{c}{ mt-lineage A-9b $(\mathrm{n}=5)$} \\
\hline 44 & 5 & 1.000 & 0.014 \\
36 & 5 & 1.000 & 0.018 \\
5 & 5 & 0.822 & 0.003 \\
2 & 2 & 0.200 & 0.001
\end{tabular}

\begin{tabular}{cccc}
\hline \multicolumn{4}{c}{ mt-lineage A-9c $(\mathrm{n}=6)$} \\
\hline 41 & 5 & 0.933 & 0.014 \\
39 & 4 & 0.800 & 0.023 \\
6 & 7 & 0.879 & 0.003 \\
4 & 5 & 0.756 & 0.004
\end{tabular}

\begin{tabular}{cccc}
\hline \multicolumn{4}{c}{ mt-lineage B-1 $(\mathrm{n}=3)$} \\
\hline 4 & 3 & 1.000 & 0.002 \\
- & 1 & - & - \\
- & 1 & - & - \\
1 & 2 & 0.333 & 0.001
\end{tabular}

\begin{tabular}{cccc}
\hline \multicolumn{4}{c}{ mt-lineage B-2a $(\mathrm{n}=12)$} \\
\hline 36 & 10 & 0.970 & 0.007 \\
42 & 10 & 0.970 & 0.013 \\
2 & 3 & 0.636 & 0.002 \\
1 & 2 & 0.228 & 0.001
\end{tabular}


POMC

SLC8A3 (NCX3)

TYR

16S

NCX1 (SLC8A1)

NFIA

POMC

SLC8A3 (NCX3)

TYR

\begin{tabular}{cccc}
6 & 3 & 0.378 & 0.003 \\
3 & 4 & 0.644 & 0.001 \\
12 & 9 & 0.978 & 0.013 \\
\hline
\end{tabular}

\begin{tabular}{cccc}
7 & 5 & 0.742 & 0.006 \\
- & 1 & - & - \\
11 & 6 & 0.848 & 0.014 \\
\hline
\end{tabular}

\begin{tabular}{llll}
8 & 5 & 0.933 & 0.008 \\
3 & 4 & 0.867 & 0.003 \\
6 & 5 & 0.933 & 0.009 \\
\hline
\end{tabular}

\begin{tabular}{cccc}
9 & 7 & 0.851 & 0.004 \\
4 & 5 & 0.361 & 0.002 \\
10 & 9 & 0.812 & 0.010 \\
\hline
\end{tabular}

\begin{tabular}{cccc}
\hline \multicolumn{4}{c}{ mt-lineage B-2b $(\mathrm{n}=8)$} \\
\hline 24 & 7 & 0.964 & 0.006 \\
33 & 7 & 0.964 & 0.014 \\
7 & 6 & 0.833 & 0.005 \\
1 & 2 & 0.125 & 0.000 \\
4 & 4 & 0.350 & 0.001 \\
3 & 3 & 0.633 & 0.002 \\
10 & 13 & 0.967 & 0.010 \\
\hline
\end{tabular}

\begin{tabular}{cccc}
\hline \multicolumn{4}{c}{ n-cluster I $(\mathrm{n}=68)$} \\
\hline 204 & 61 & 0.996 & 0.026 \\
218 & 57 & 0.994 & 0.049 \\
19 & 26 & 0.875 & 0.005 \\
11 & 16 & 0.493 & 0.002 \\
29 & 26 & 0.784 & 0.006 \\
9 & 10 & 0.593 & 0.002 \\
34 & 58 & 0.935 & 0.014 \\
\hline
\end{tabular}

\begin{tabular}{cccc}
\hline \multicolumn{4}{c}{ n-cluster II (n = 56) } \\
\hline 176 & 53 & 0.997 & 0.018 \\
192 & 47 & 0.994 & 0.037 \\
12 & 13 & 0.483 & 0.002 \\
6 & 8 & 0.486 & 0.001 \\
24 & 24 & 0.849 & 0.007 \\
12 & 12 & 0.577 & 0.002 \\
33 & 43 & 0.907 & 0.016 \\
\hline
\end{tabular}




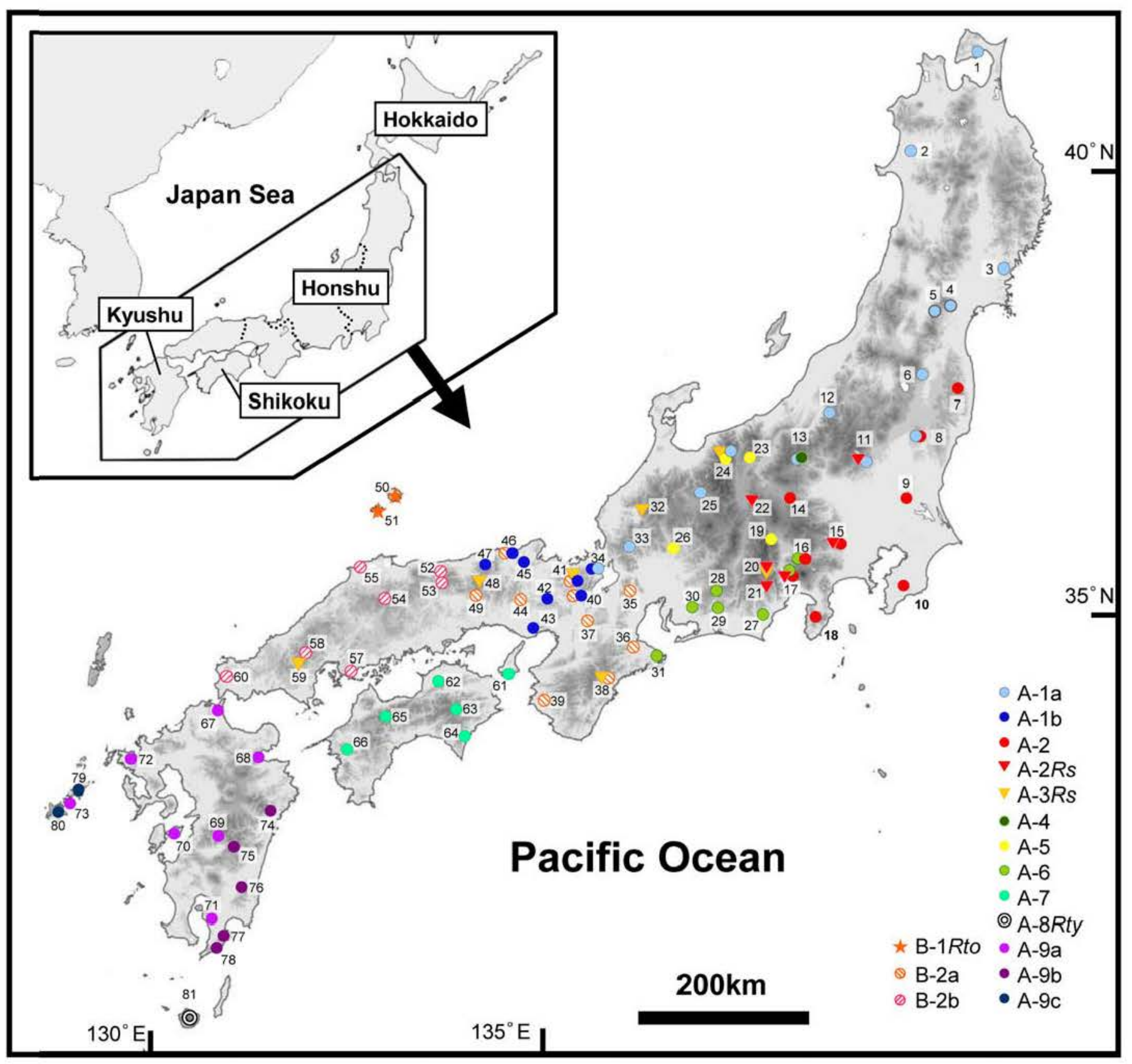

Fig. 1 


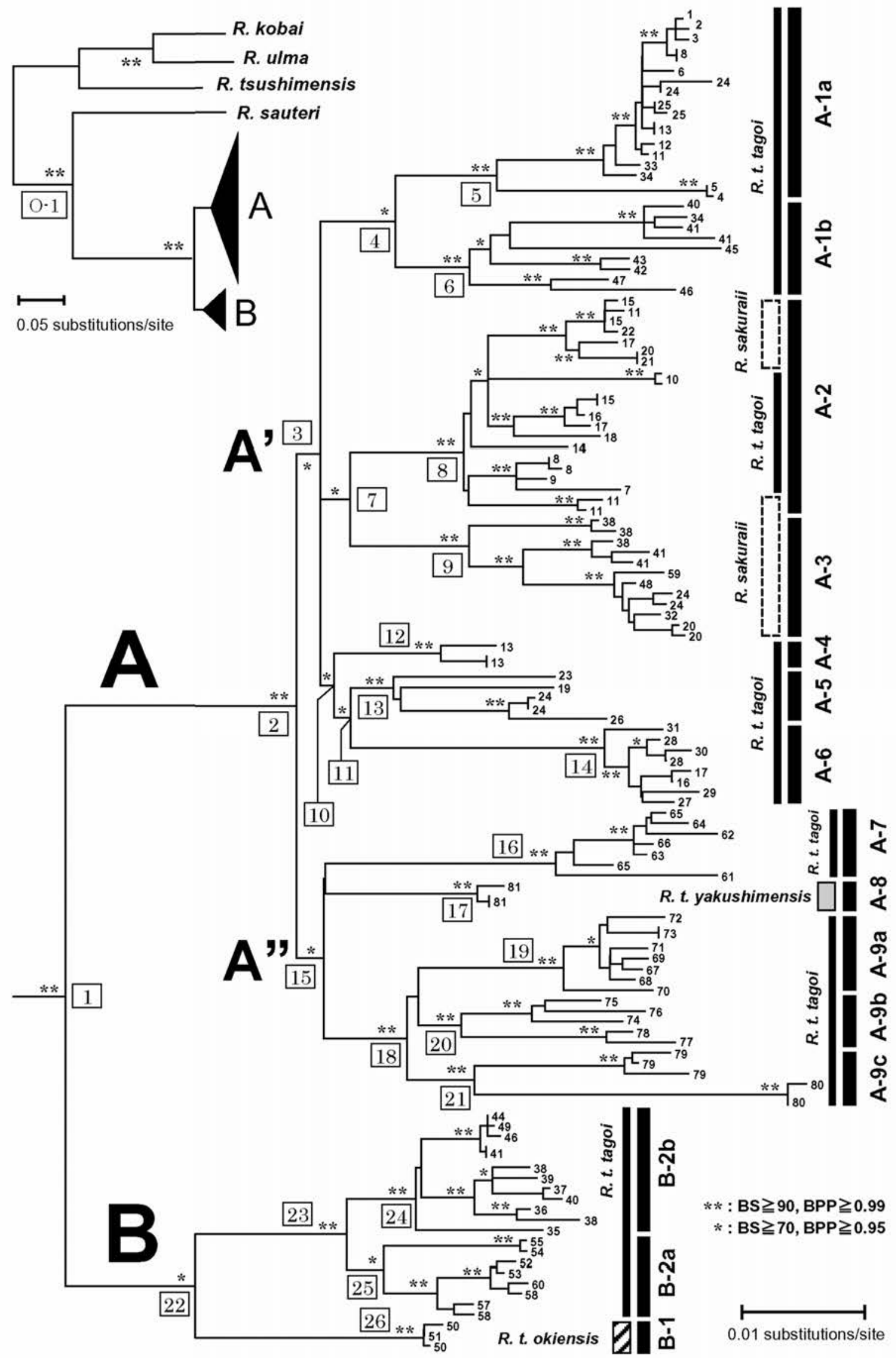

Fig. 2 


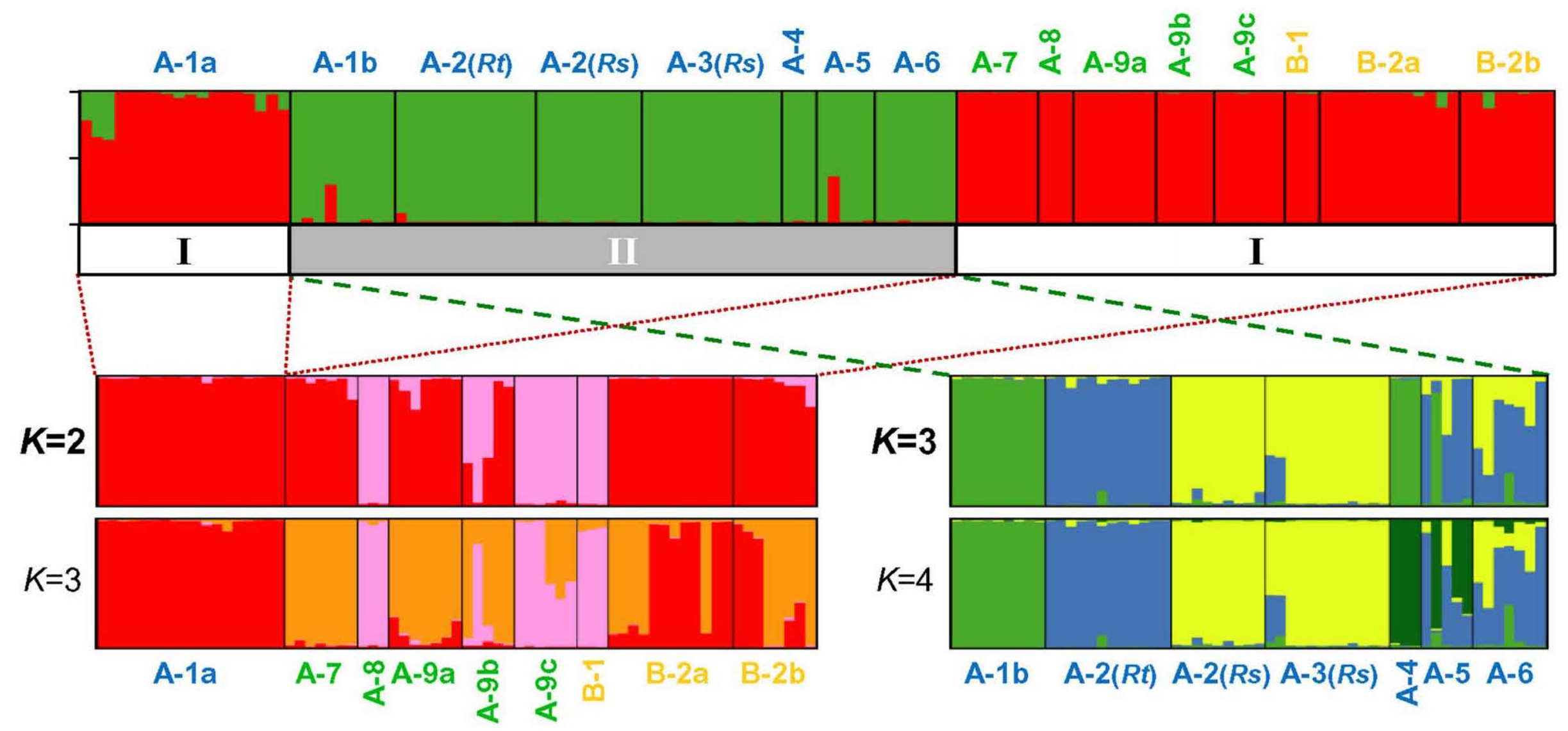

Fig. 3 


\section{1: ILS}

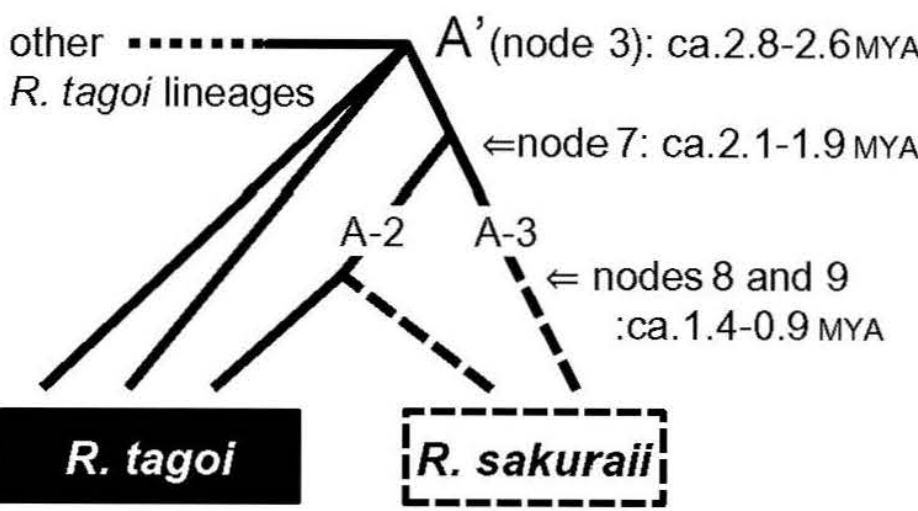

\section{2: introgression}

a

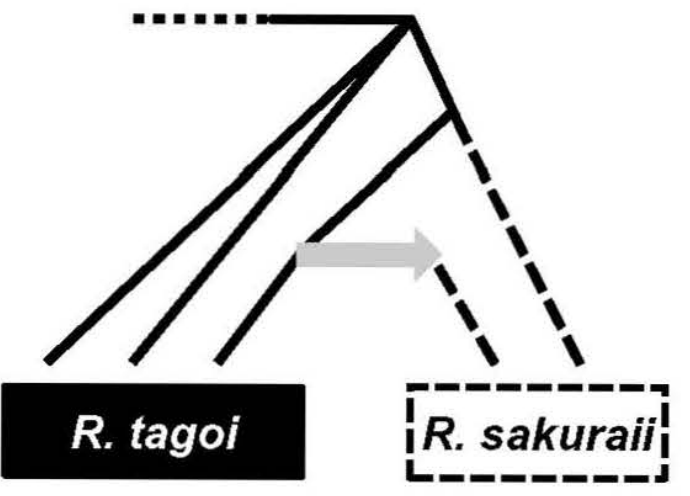

b

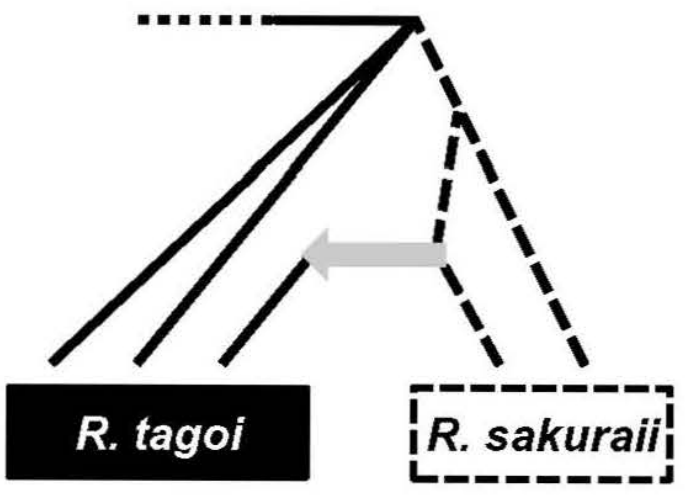

Fig. 4 


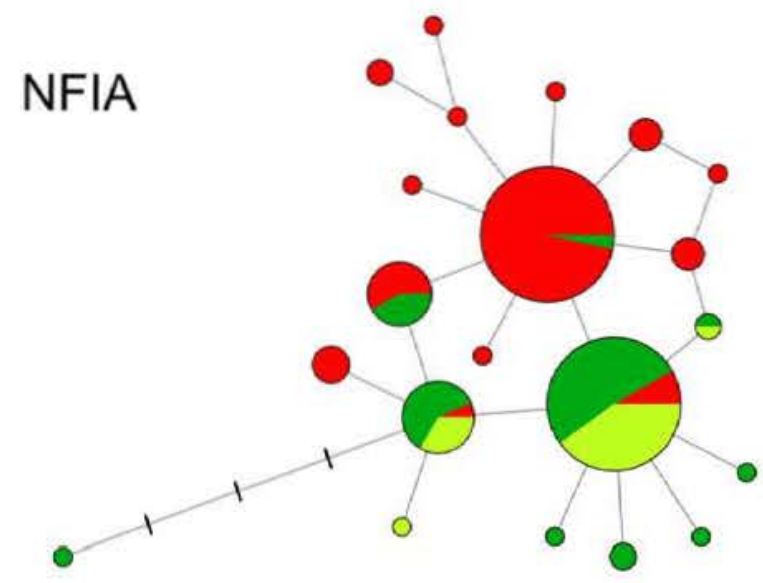

NCX1
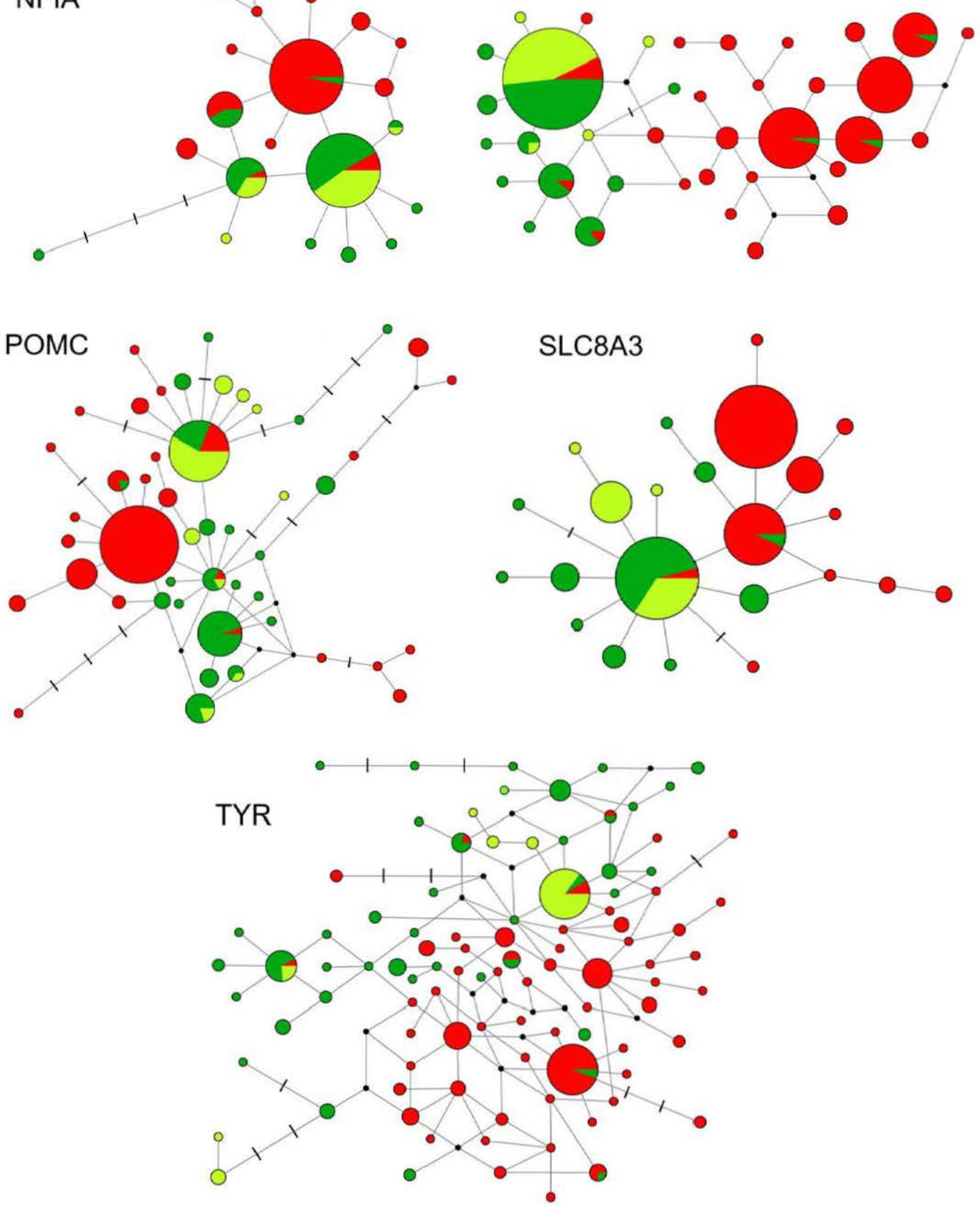

Fig. S1 

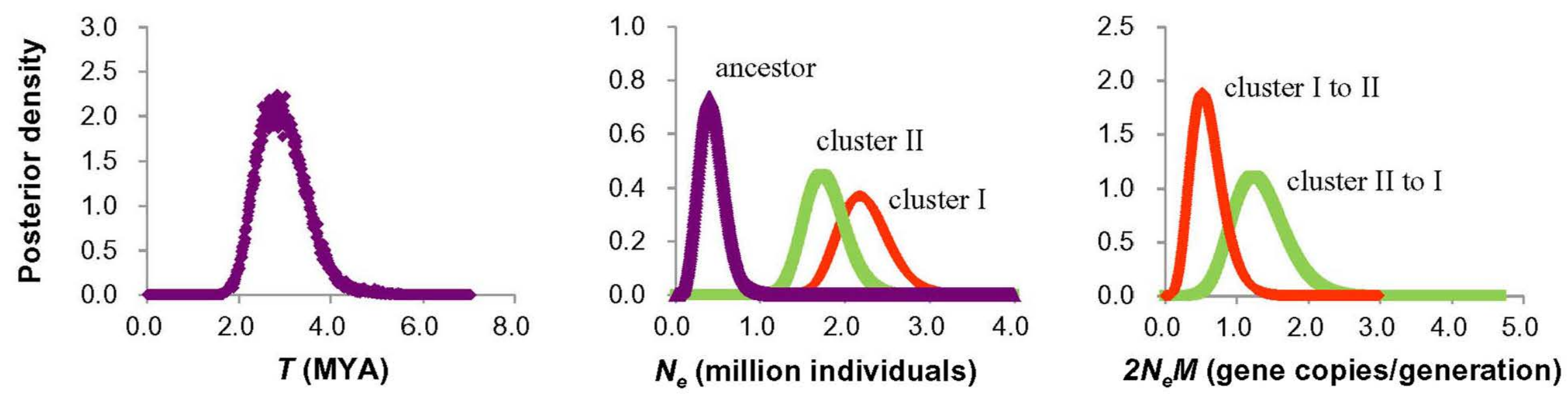

Fig. S2 

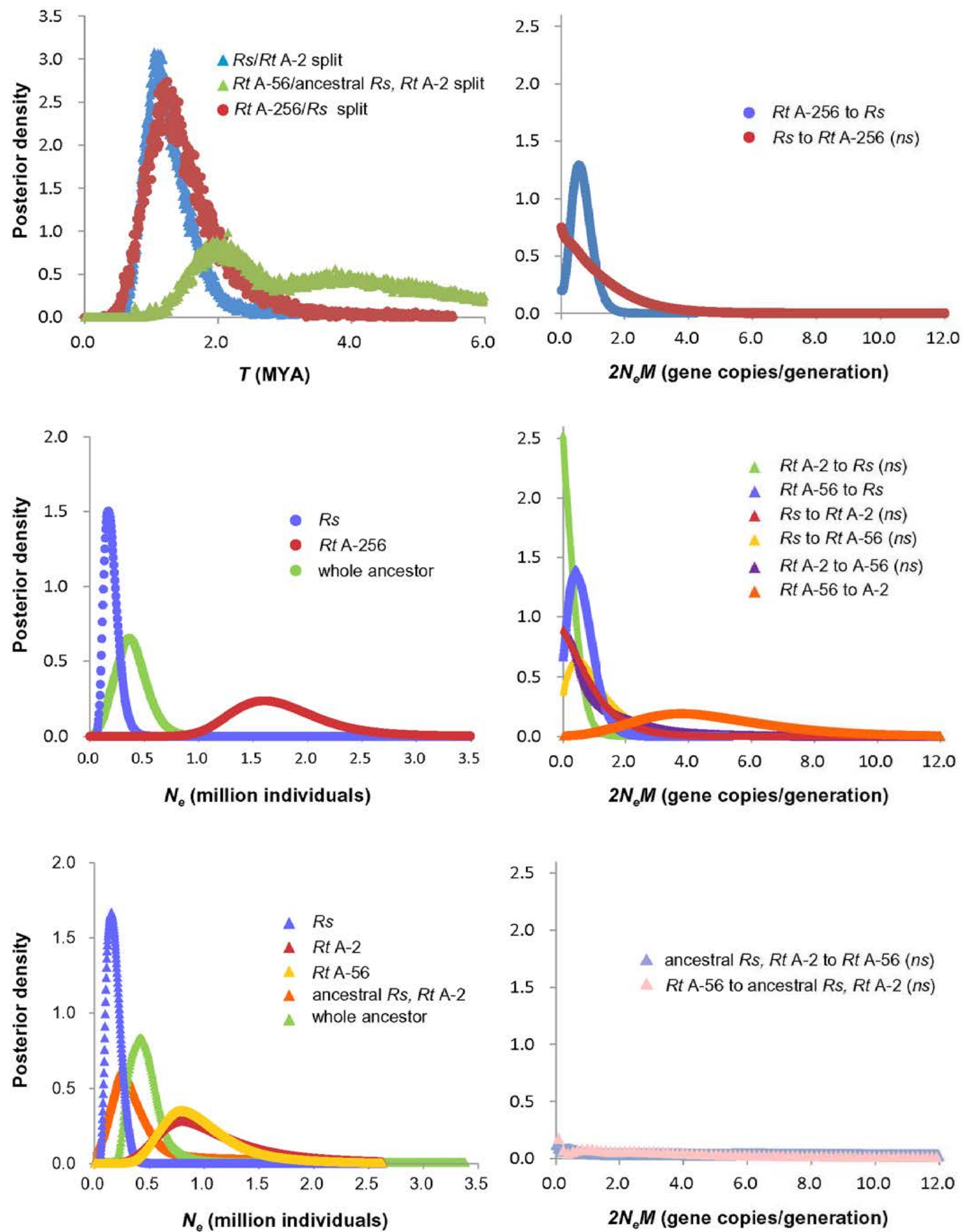

Fig. S3 

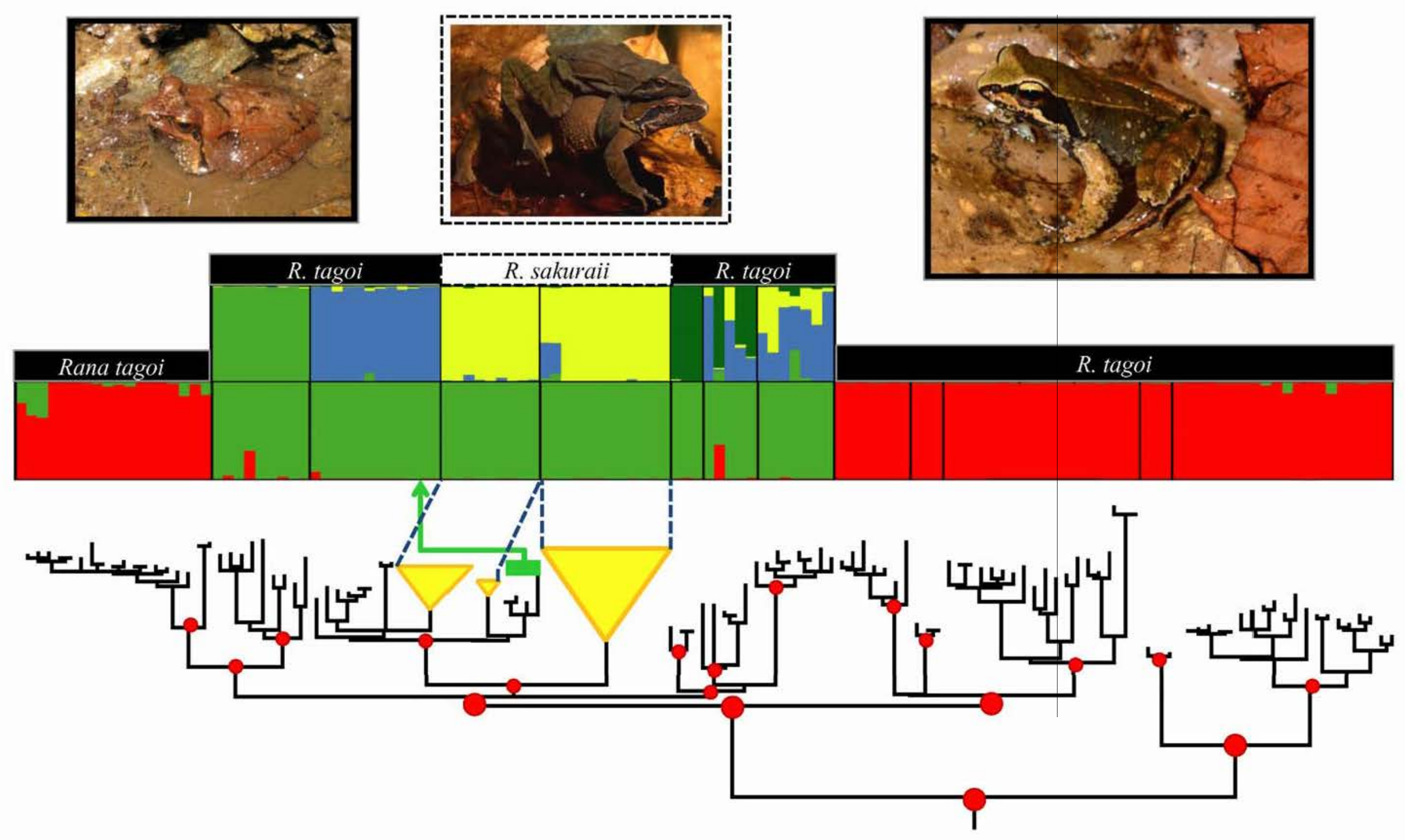

Graphical abstract 